\title{
Images suffisantes - images efficaces : à propos de pinakes figurés dans la céramique attique
}

Sufficient images - effective images. About pinakes represented on attic vases

\section{Vasiliki Zachari}

\section{OpenEdition}

1 Journals

Édition électronique

URL : http://journals.openedition.org/imagesrevues/9290

DOI : 10.4000/imagesrevues.9290

ISSN : 1778-3801

Éditeur :

Centre d'Histoire et Théorie des Arts, Groupe d'Anthropologie Historique de l'Occident Médiéval, Laboratoire d'Anthropologie Sociale, UMR 8210 Anthropologie et Histoire des Mondes Antiques

\section{Référence électronique}

Vasiliki Zachari, «Images suffisantes - images efficaces : à propos de pinakes figurés dans la céramique attique », Images Re-vues [En ligne], Hors-série 9 | 2020, mis en ligne le 01 décembre 2020, consulté le 16 mars 2021. URL : http://journals.openedition.org/imagesrevues/9290 ; DOl : https:// doi.org/10.4000/imagesrevues.9290

Ce document a été généré automatiquement le 16 mars 2021.

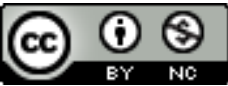

Images Re-vues est mise à disposition selon les termes de la Licence Creative Commons Attribution Pas d'Utilisation Commerciale 4.0 International. 


\section{Images suffisantes - images efficaces : à propos de pinakes figurés dans la céramique attique}

Sufficient images - effective images. About pinakes represented on attic vases

Vasiliki Zachari

\section{NOTE DE L'AUTEUR}

Toutes les dates sont avant J.-C. sauf indication contraire.

Pour leur aide précieuse lors de différentes étapes de cet article, je remercie chaleureusement L. Chadezon, T. Galoppin, T. Golsenne, F. Lissarrague, C. Mazet, S. Montel, M. Vantses, P. Zagefka et last but not least les éditrices G. Puma et S. Wyler. 
1 Les images peintes sur les vases de terre cuite, fabriquées dans le quartier du Céramique d'Athènes aux $\mathrm{VI}^{\mathrm{e}}$ et $\mathrm{V}^{\mathrm{e}} \mathrm{s}$. ouvrent quelques possibilités de contribuer à l'analyse de l'image dans l'image $^{1}$. Il s'agit plus précisément des motifs variés, peints en silhouette, c'est-àdire en vernis noir et en miniature dans l'image d'ensemble, sur des objets d'utilités diverses, qui deviennent à leur tour des supports d'images. Ainsi le bouclier, " objet théorique », est l'exemple par excellence de la créativité et de

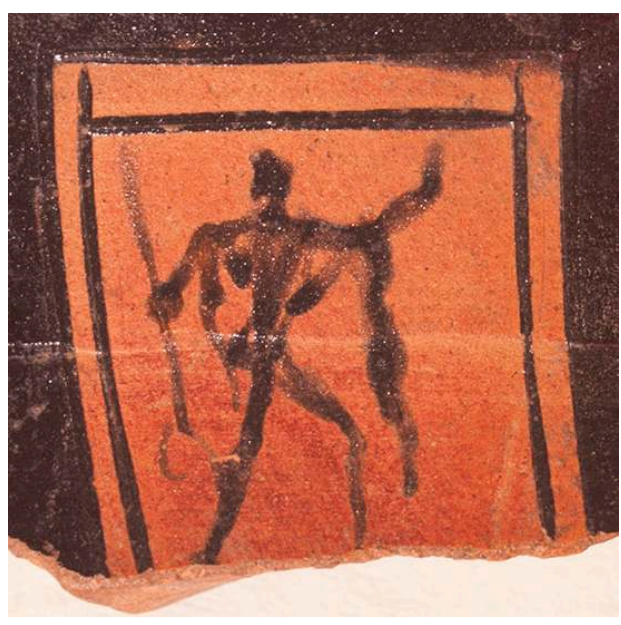
l'ingéniosité des peintres ${ }^{2}$, mais aussi les autels et les pinakes, pour donner quelques exemples de la série de supports d'images. Le pinax n'est pas un objet cultuel quelconque : il est porteur d'image et pour les Grecs, un culte sans image est inconcevable ${ }^{3}$. Le terme "pinax" recoupe plus précisément deux catégories distinctes, les tableaux funéraires et les tableaux votifs. Le pinax est une « image-objet ${ }^{4}$ », destinée à une pratique rituelle précise, celle de l'offrande dans le cas des tableaux votifs, qui constituent l'objet de cette étude, afin de demander un bienfait ou remercier les dieux pour un service rendu, créant ainsi un lien direct entre le dédicant et la divinité. En juxtaposant les représentations figuratives à la réalité archéologique, nous constatons une dissociation remarquable, puisque les choix iconographiques ne sont pas toujours en accord avec les preuves matérielles. Dans cet article, il sera question d'aborder l'aspect figuratif des images de pinakes, leurs contextes et leur emplacement dans la scène et surtout leur contenu, les choix faits par les peintres et l'impact de ces images dans le contexte cultuel représenté, ainsi que l'interaction entre l'objet - le pinax et l'image portée -, et l'image d'ensemble. Dans un premier temps, nous allons traiter le pinax selon le champ lexical et archéologique pour aborder par la suite la façon de le dessiner sur les vases. Dans la deuxième partie, nous allons approfondir l'étude en rassemblant les pinakes par motif figuré afin de comprendre pourquoi les peintres les représentent. L'objectif est de déchiffrer quelles sont la fonction et de la nature des images portées sur les tableaux votifs : "à quoi servent-elles? Et cela peut-il nous apprendre quelque chose sur ce qu'elles sont ${ }^{5}$ ?»

\section{Pinax : termes, realia et représentations}

2 Le terme pinax (pluriel pinakes), déjà attesté dans l'Odyssée ${ }^{6}$ et l'Iliade $e^{7}$, désigne en général un objet plat ${ }^{8}$ et plus précisément une planche de forme rectangulaire destinée à devenir support d'écriture (une tablette), ou de peinture (un tableau) ${ }^{9}$. Graphein, d'ailleurs, signifie en grec ancien à la fois "écrire " et "dessiner » ${ }^{10}$. Adopté par l'érudition moderne, le mot pinax renvoie surtout, en ce qui concerne l'Antiquité, aux œuvres exécutées sur une plaque de forme rectangulaire et peinte, en bois ou en terre cuite $^{11}$. Mais il s'agit en fait d'un terme générique encadrant d'autres catégories d'objets, sans indication sur la technique et la matérialité de l'objet, précisées par des adjectifs qualificatifs, comme le montrent les textes littéraires et surtout les inscriptions administratives de Délos. Ces témoignages fournissent une autre 
information importante quant à leur fonction, car la documentation distingue deux catégories de pinakes selon le sujet qu'ils portent: les pinakes eikonikoi et les pinakes anathematikoi, les premiers étant des portraits peints ${ }^{12}$ et les seconds des tableaux votifs ${ }^{13}$. Ces derniers ont été retrouvés in situ dans les sanctuaires où ils ont été déposés et dédiés.

3 Les données à notre disposition sont hétéroclites en termes géographiques, chronologiques, ainsi que matériels. Elles fournissent, cependant, une image représentative des moyens et des techniques de fabrication des pinakes dans l'Antiquité. La matière choisie et la technique appliquée déterminent également l'image portée. Les techniques du repoussé ou du moule, utilisées notamment pour les ex-voto en métal ou les tablettes votives en terre cuite, favorisent la création d'images abrégées, avec peu d'éléments figuratifs, faisant d'elles des objets sériels - fabriqués rapidement et en abondance à partir du même modèle sans que soit porté un intérêt particulier aux détails de l'image, parfois rendus avec l'utilisation des couleurs ${ }^{14}$. La peinture, d'autre part, permet la création d'objets plutôt uniques et élaborés en apportant plus de précisions à l'image, ainsi qu'un aspect plus vivant avec l'utilisation de couleurs, comme l'attestent les pinakes en bois ${ }^{15}$. De plus, pour ce qui est de la peinture sur terre cuite, la cuisson du support de l'image au four permet la préservation de ces images ainsi transformées en "baked prayers $»^{16}$. Leur forme est surtout rectangulaire et nous trouvons moins souvent des objets votifs en forme de carré ou de naiskos (temple en miniature). Quant à leur orientation notamment pour les objets rectangulaires, elle est généralement horizontale, ce qui permet de développer une scène avec plusieurs participants et des éléments architecturaux ou naturels, ou même des scènes étendues sur plusieurs registres parallèles. L'orientation verticale semble plus propice afin d'isoler et mettre en valeur un seul élément ou une seule figure, particulièrement pour les ex-voto en métal, mais aussi en terre cuite. Les images portées sont d'une grande variété : représentations des divinités ou des héros vénérés, des donateurs isolés ou en groupe, des sujets mythologiques, des rituels ou d'autres thématiques, tout comme des objets et des animaux. Ces pinakes sont parfois porteurs d'inscriptions dédicatoires peintes ou gravées ${ }^{17}$, qui leur confèrent ainsi un caractère plus personnalisé, et plus rarement de signatures des leurs créateurs ${ }^{18}$, proclamant ainsi leur art placé sous la protection divine. Enfin, des trous de suspension sont préservés pour les objets destinés à être accrochés à l'aide d'une ficelle ou d'une lanière de cuir. 


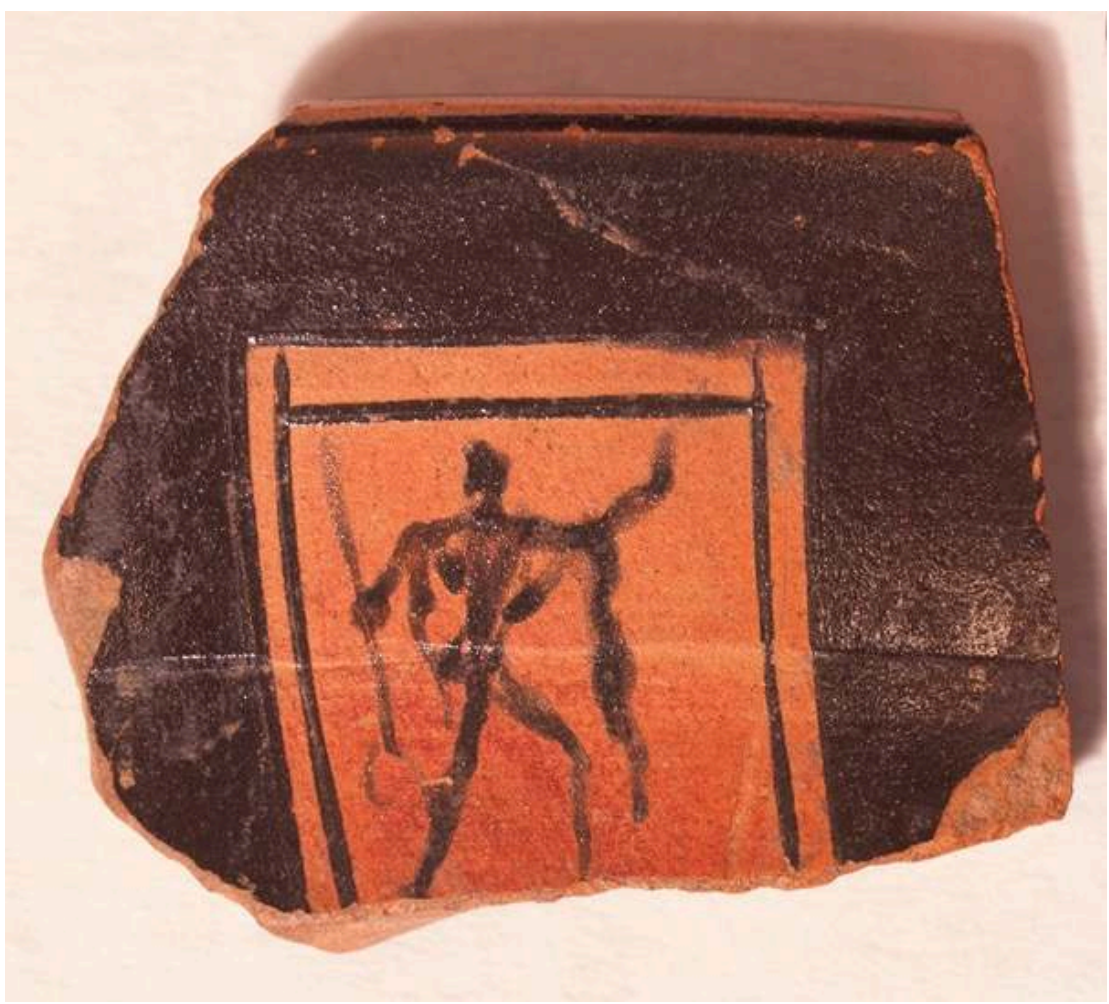

Fragment de rhyton à figures rouges (f.r.) Bryn Mawr, Bryn Mawr College P 913 (BAPD 1621).

(C) Bryn Mawr College

4 La représentation des pinakes sur la céramique est facile à repérer : il s'agit d'un petit carré rouge figurant en hauteur dans le champ de la scène et portant en général une image peinte en noir. Le corpus consiste en une cinquantaine de vases à figures rouges, majoritairement de vases attiques, datant d'une période qui s'étend de 480 jusqu'au $\mathrm{IV}^{\mathrm{e}}$ siècle environ ${ }^{19}$. Le fragment d'un rhyton de la collection de Bryn Mawr College (fig. 1) nous permet de regarder de plus près et attentivement ce détail figuratif afin de comprendre la technique de son exécution. Tout d'abord, une ligne de relief, rendue avec du vernis plus épais, contourne le pinax marquant ainsi la limite entre les deux zones décoratives, en noir et rouge respectivement pour la scène principale, ici perdue, et l'image dans l'image, la mettant ainsi en évidence. Cet effet est souligné davantage au moyen du cadre rendu par des traits fins à l'intérieur du tableau votif ${ }^{20}$. Le sujet représenté est une figure humaine en mouvement, comme l'indiquent les jambes écartées et les bras agités. Le menton semble prolongé, ce qui signale probablement une barbe; on déduit ainsi qu'il s'agit d'une figure masculine, comme semblent le confirmer l'objet oblong qu'il tient dans la main droite, probablement un bâton, et le pan de vêtement qui pend d'un bras à l'autre. Ces éléments sont communs dans l'iconographie des comastes ${ }^{21}$ sur les vases ${ }^{22}$. Néanmoins, ni les dimensions de la figure, ni le rendu ne permettent de confirmer cette proposition avec certitude ou d'aller plus loin dans l'identification de l'homme ${ }^{23}$. En outre, le dessin hâtif de l'image rend impossible son attribution à un peintre.

Dans ce corpus d'images, les pinakes peints dans le champ de l'image sont surtout rectangulaires, parfois carrés ${ }^{24}$, et l'on connaît peu de cas nous les montrant en forme de naiskos ${ }^{25}$. Ils sont de dimensions réduites d'un à deux centimètres, ce qui ne permet 
pas d'élaborer une scène complète, mais plutôt de peindre un seul élément, comme une image en version abrégée. Les peintres favorisent l'orientation verticale avec une figure unique, tandis que l'orientation horizontale, plus rare, permet d'ajouter plusieurs détails ${ }^{26}$. Très régulièrement, les peintres prennent le soin d'encadrer les images portées dans les pinakes avec plus ou moins de méticulosité. Les pinakes sont très souvent peints dans le champ de l'image comme un objet attaché, parfois avec la tige d'accrochage représentée ${ }^{27}$; d'autres fois ils sont représentés suspendus à un arbre ${ }^{28}$ ou à une branche ${ }^{29}$ et plus rarement portés à la main ${ }^{30}$. Dans la plupart des cas, un seul pinax est peint dans une scène, mais on peut en compter parfois plusieurs ${ }^{31}$. Le contexte de la scène où l'on voit les pinakes est majoritairement rituel et plus rarement il s'agit de l'atelier du forgeron ${ }^{32}$. La matérialité de ces objets n'est pas indiquée sur le vase. Il est néanmoins plausible de supposer qu'il s'agisse de représentations de tableaux votifs peints en terre cuite; on aurait alors affaire à une image en abyme, ainsi qu'à une allusion autoréférentielle à leur production dans l'atelier du potier ${ }^{33}$. Enfin, concernant les images portées, nous allons aborder par la suite le contenu des tableaux votifs figurés dans le champ de l'image qui sont ici regroupés et analysés plus précisément selon le motif représenté, en trois ensembles : figures divines et humaines, animaux et êtres hybrides et, enfin, motifs abstraits et pinakes vides, sans aucune figuration, dans le but de comprendre les choix opérés et les jeux d'images créés par les peintres quand ils représentent des pinakes sur les vases.

\section{Hommes et dieux}




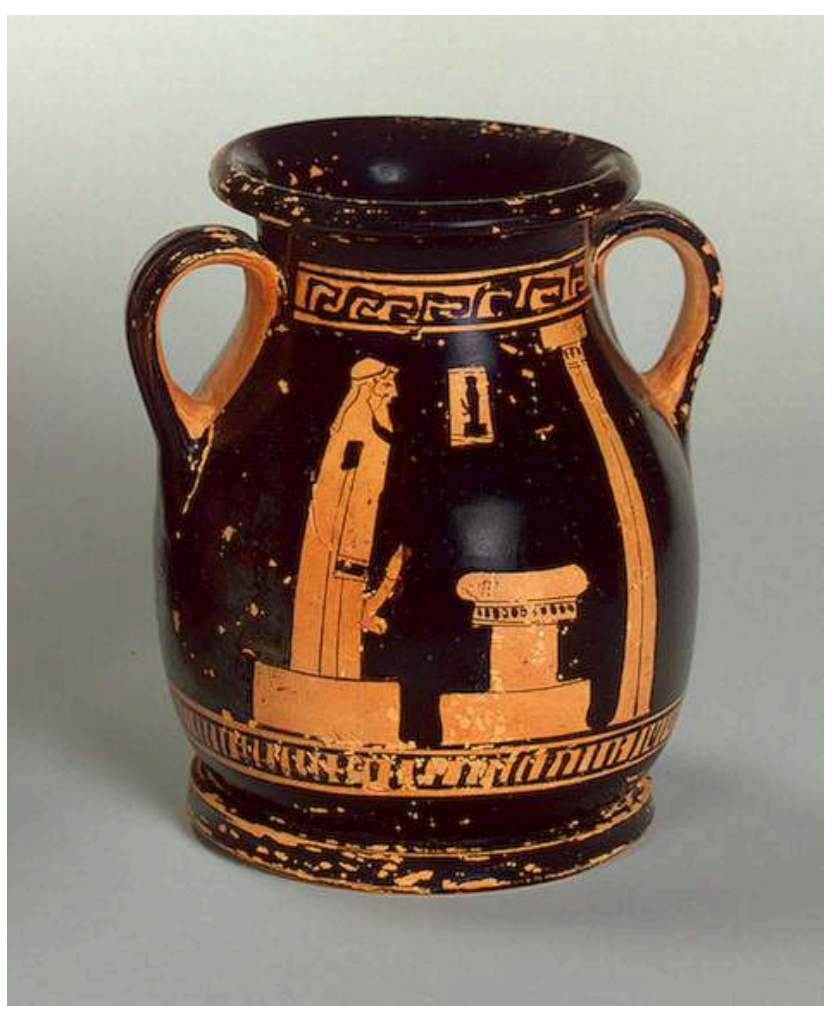

Pélikè à f.r., St. Petersburg, Musée de l'Ermitage B 4515 attribué au peintre de Bowdoin, vers 470 (BAPD 9017636).

(C) Musée de l'Ermitage

Nous commençons par la figure divine la plus souvent représentée et la plus facilement reconnaissable dans ce corpus d'images: le dieu Hermès et plus précisément sous forme de statue (hermès ou pilier hermaïque). Sur une pélikè à Saint-Pétersbourg (fig. 2) trois éléments architecturaux composent la scène définissant l'espace sacré : une colonne dorique à droite, un autel sur degrés au centre et un hermès également sur une base à gauche. Habituellement ce dernier est identifié par sa verticalité, son sexe érigé et sa tête barbue, seules caractéristiques anthropomorphes, et il est souvent combiné avec un autel ${ }^{34}$. Parfois un vêtement couvre son torse ${ }^{35}$, comme sur ce vase. Un nouveau détail s'ajoute à cette composition d'un espace sacré et attire notre attention : il s'agit du tableau votif qui s'inscrit dans la prolongation de l'autel créant ainsi un troisième axe vertical dans la scène. L'image figurée sur le tableau votif ne passe pas inaperçue. Des traits fins à la partie supérieure et inférieure du tableau encadrent l'image d'un pilier hermaïque, peint en silhouette, qui fait face à la statue du dieu dans la scène principale. L'image dans l'image opère dans cet exemple comme un négatif avec l'inversion des couleurs, - noir sur rouge pour le pinax et rouge sur noir pour la scène principale - l'inversion de l'orientation et le jeu de proportions entre les deux représentations de la statue, en petite et grande échelle respectivement. La préférence pour cet élément peut s'entendre dans la mesure où il s'agit d'un motif très codifié et très facile à exécuter, tel qu'on le constate parfois comme exercice d'esquisse sur la sculpture $^{36}$. Ce choix peut être interprété également selon le contexte de la scène. En effet, l'image miniature du pilier hermaïque figurant dans les tableaux votifs est récurrente sur d'autres vases, dans des scènes similaires d'espace sacré sans présence humaine, uniquement avec des éléments architecturaux ou naturels, ou dans des 
scènes avec des figures humaines en contexte d'offrande ${ }^{37}$ ou d'installation d'hermès ${ }^{38}$. À chaque fois elle est juxtaposée avec la grande image de la statue selon le même modèle de disposition, l'une faisant face à l'autre comme en miroir ${ }^{39}$. Par cette association qui fait écho entre la statue et sa représentation, le peintre aboutit au dédoublement de l'image divine, créant ainsi une mise en abyme. Néanmoins, son ingéniosité va plus loin, puisque l'hermès peint dans le pinax est une image au troisième degré, l'image du dieu sous la forme de sa statue dans la scène principale opérant déjà comme une image dans l'image ${ }^{40}$.

Figure 3

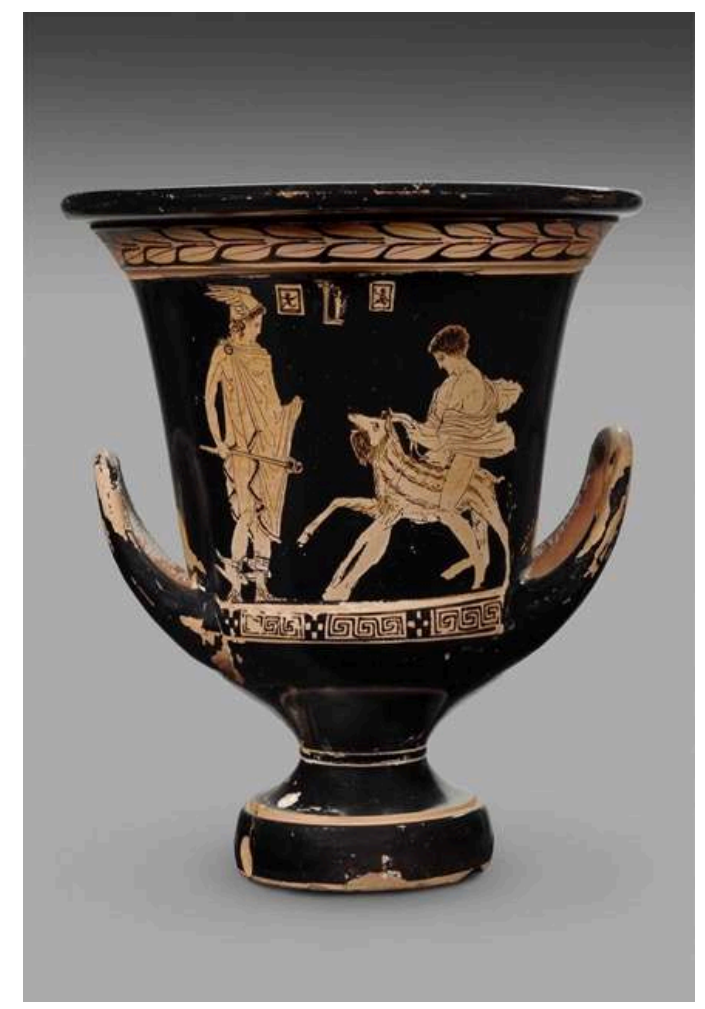

Cratère en calice à f.r., Boston Museum of Fine Arts 03.796, manière du peintre des Baigneuses, vers 420 (BAPD 41010).

(C) Museum of Fine Arts

$7 \quad$ Un procédé figuratif de dédoublement de l'image divine assez particulier est attesté en contexte rituel sur un cratère à Boston (fig. 3), où le dieu Hermès est présentifié simultanément sous deux formes différentes par rapport au vase précédent : avec le dieu lui-même et sa représentation sous forme de statue en miniature dans le champ de l'image, non pas peint dans les pinakes cette fois-ci, mais comme une sorte d'objet votif en trois dimensions ${ }^{41}$. L'association figurative d'une divinité avec sa statue n'est pas très courante dans la céramique attique et ce vase constitue une exception originale ${ }^{42}$. La scène d'un caractère sophistiqué est structurée comme un acte rituel en version abrégée. Un jeune homme amène un animal devant le dieu Hermès. La présence divine précise le destinataire du sacrifice, restant souvent inconnu dans des scènes de sacrifice avec autel qui sont plutôt génériques ${ }^{43}$. Par son aspect anthropomorphe et son naturalisme, Hermès est présent dans l'image en épiphanie, probablement invisible au jeune homme comme en attestent leurs regards qui ne se croisent pas, mais toutefois visible par le spectateur ${ }^{44}$. De cette façon, le peintre met en valeur l'acceptation du 
sacrifice par le dieu. Cette relation don / contre-don est davantage accentuée par la présence des objets traces de la piété humaine dans le champ de l'image: les deux pinakes avec des figures humaines qui encadrent la représentation du dieu en micrographie, signifiant ainsi la matérialité du dieu dans l'image ${ }^{45}$. L'offrande de l'image divine est une pratique courante dans les sanctuaires, comme l'attestent les nombreux objets votifs. En ce qui concerne les pinakes trouvés dans des sanctuaires et l'image d'Hermès plus précisément, nous constatons la présence du dieu, soit isolée ${ }^{46}$, soit avec d'autres divinités ${ }^{47}$ et l'absence du motif du pilier hermaïque pourtant abondant sur les vases. Cet écart entre réalité rituelle et figurative est probablement dû à l'envie des peintres de créer des jeux d'images avec les éléments à leur disposition, à savoir l'image d'Hermès dans des versions variées, une divinité propice à ce type de jeux graphiques qui nous permettent de comprendre comment «la présence et la représentation sont les deux outils conceptuels qui construisent le rapport des fidèles au divin $»^{48}$.

Figure 4

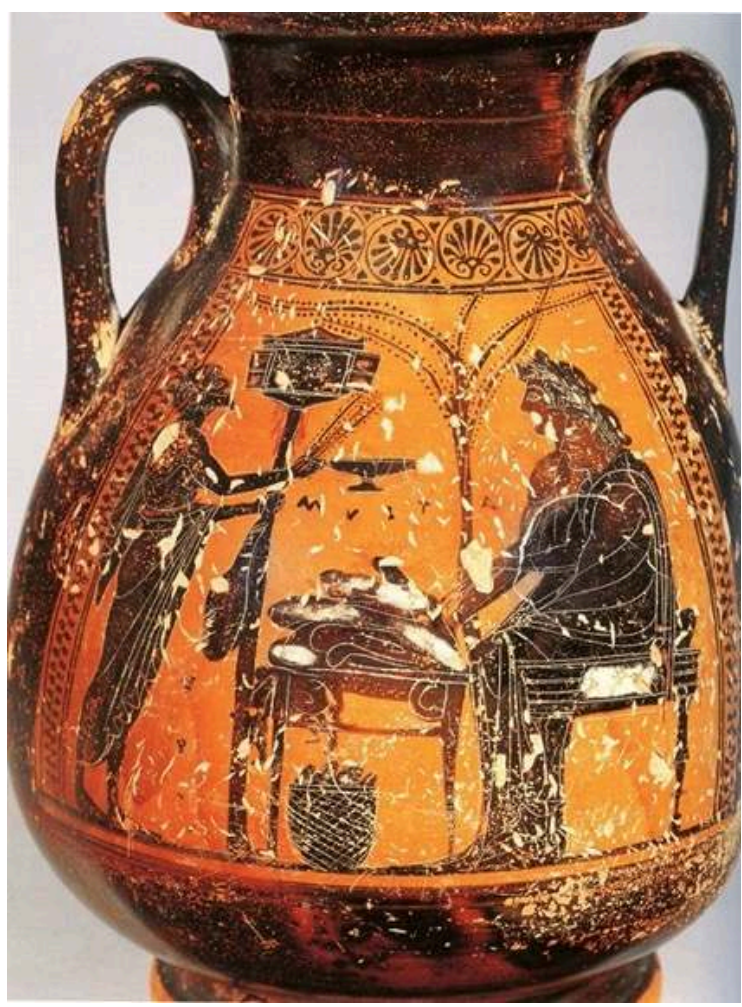

Pélikè à figures noires (f.n.), Naples, Museo Archeologico Nazionale 81083, près du peintre de Rycroft, 520-500 (BAPD 301859).

(C) Archivio fotografico della Soprintendenza Archeologica delle province di Napoli e Caserta. 


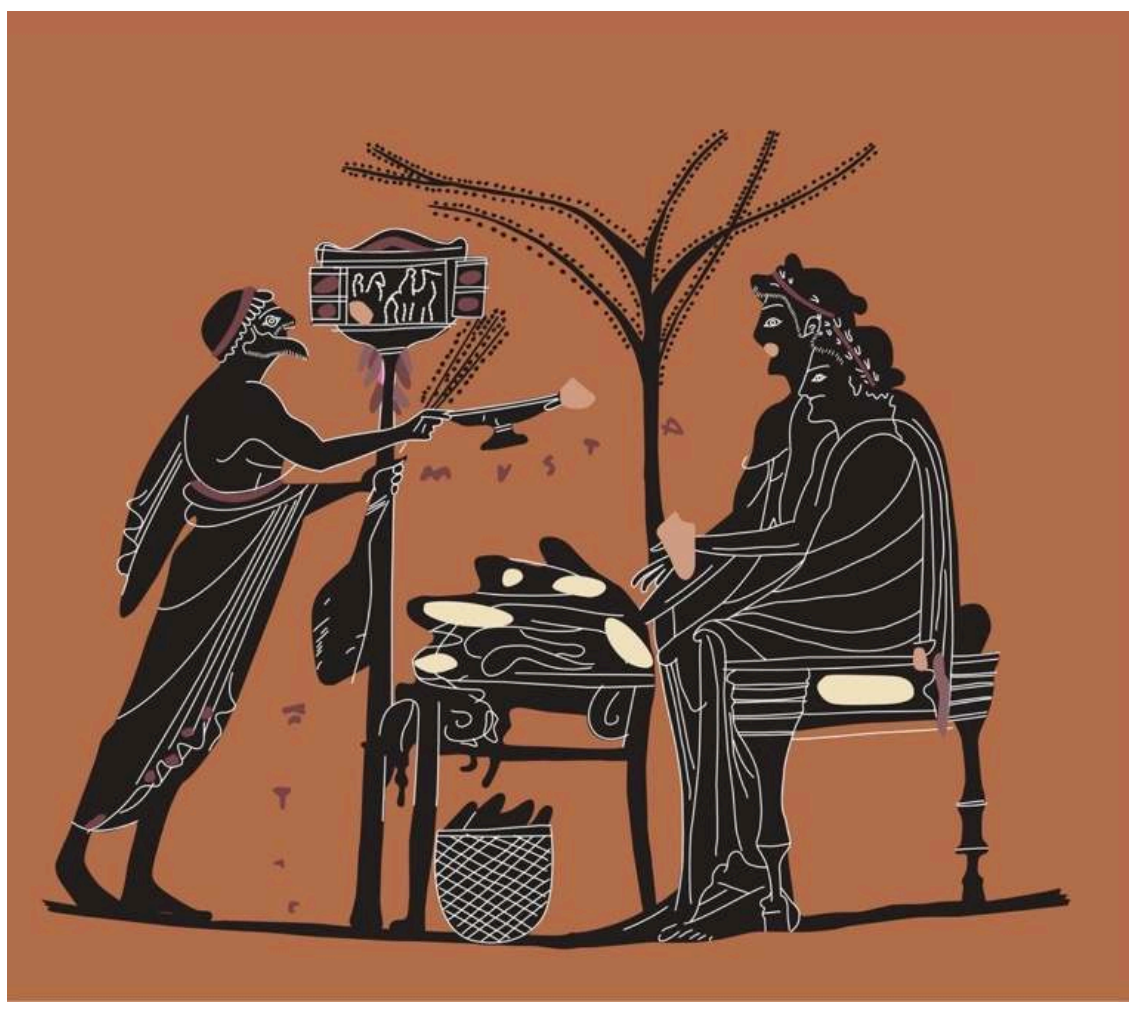

Dessin vectoriel.

䢻 Ludivine Chazalon

8 Un troisième vase, un hapax dans le répertoire attique, nous permet d'aborder un autre type de fonctionnement de l'image divine telle qu'elle est représentée sur un pinax. La pélikè du musée de Naples (fig. 4) fait exception dans la série des pinakes à cause de la technique - à figures noires - et du support du tableau votif. Deux jeunes hommes couronnés sont figurés assis à droite dans la scène principale devant une table chargée d'objets divers ${ }^{49}$ et un panier, contenant probablement de la nourriture, posé par terre ; un arbre se dresse au second plan. De l'autre côté de la table, un homme barbu tient dans la main droite une coupe et une branche et, dans la main gauche, un morceau de viande. Derrière sa main surgit un pilier surmonté d'un pinax en forme de naiskos avec deux battants ouverts de part et d'autre, tel un triptyque, et orné de bandelettes rouges. Sur le pinax central (fig. 4bis) deux cavaliers sont peints en rehaut blanc, tandis que les motifs qui l'encadrent, figurant par deux sur chaque battant, restent indéchiffrables. Enfin, l'inscription MYSTA au centre de la scène, entre les hommes, nous laisse très perplexe $e^{50}$. Le manque de parallèles ne nous permet pas d'aller très loin dans l'interprétation de cette scène unique et très complexe. Néanmoins, quelques éléments parlants permettent de la contextualiser, d'identifier les cavaliers et d'avancer quelques hypothèses sur l'effet de l'image dans l'image. Le contexte rituel de la scène nous semble incontestable, comme le révèle la présence des objets tenus par l'homme barbu à gauche de la scène: la coupe pour faire la libation, les branches tenues et la bandelette portée à sa tête pour souligner le caractère festif, le morceau de viande - la cuisse désossée, un gigot mou, qui correspond plus précisément à une part d'honneur de l'animal sacrifié ${ }^{1}$ - et le pinax. En vertu de quelle occasion ces éléments souvent dissociés entre eux dans l'imagerie attique sont-ils ici exceptionnellement 
assemblés ? La présence de la table offre une piste féconde à explorer. Objet mobile souvent représenté en contexte de banquet, la trapeza est également un instrument du sacrifice partageant des rapports homologues avec l'autel ${ }^{52}$. Elle figure souvent sur des vases comme support pour la découpe de l'animal sacrificiel ${ }^{53}$, ainsi que pour déposer des offrandes notamment dans le cadre de certains rituels, dont les théoxénies ${ }^{54}$. Ce rituel consiste en l'accueil temporaire des dieux et de héros à une table préparée par les hommes où se construit l'échange sous forme d'hospitalité. Peu de représentations figurées sont connues et elles sont toutes remarquables, montrant souvent les Dioscures en mouvement. Ainsi, nous pourrions identifier comme les destinataires de ces offrandes les deux cavaliers représentés dans le pinax, c'est-à-dire les jumeaux divins de la mythologie $e^{55}$. Par conséquent, les deux images, la scène principale et celle du tableau votif, fonctionnent en complémentarité et en corrélation, la compréhension et l'identification de l'une permettant l'explication de l'autre.

En ce qui concerne les figures humaines représentées dans les tableaux votifs, la série la plus abondante dans le corpus, la grande difficulté à laquelle nous nous heurtons est celle de l'identification et de la surinterprétation. Le regroupement de ces images s'avère une affaire délicate puisque l'individuation des personnages et la reconnaissance des objets tenus ou manipulés est souvent impossible. Dans cette série d'images, nous nous permettrons d'avancer quelques hypothèses en les classant en deux groupes distincts : les figures immobiles et en mouvement.

Figure 5

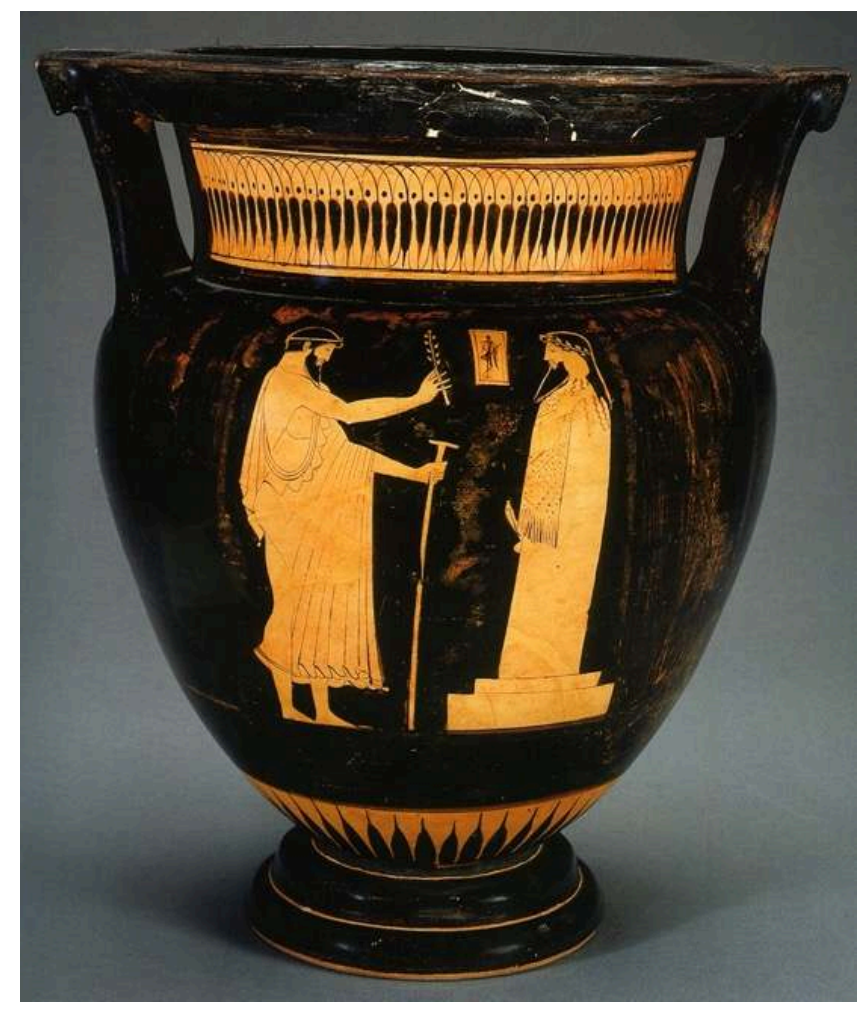

Amphore à f.r., Christies' New York 2000 n 84, attribué au peintre du Porc, 480-470 (BAPD 275736).

(C) 2000 Christie's Images Limited

Nous commençons par les images humaines immobiles, et plus précisément par une figure relativement simple d'interprétation. Sur un cratère du marché des Antiquités 
(fig. 5) un homme barbu appuyé sur son bâton tend une branche vers un pilier hermaïque ${ }^{56}$. Dans le champ de l'image, la figure représentée dans le pinax reproduit certains éléments caractéristiques comme le bâton du citoyen et le vêtement qui le couvre. En plus, il est orienté vers l'hermès comme pour répéter et ainsi accentuer l'adresse de la figure principale vers la statue du dieu. Même si la posture du corps se différencie entre les deux figures, ce qui ne nous permet pas de parler d'une reproduction fidèle de l'image principale, il nous semble que le peintre du Porc a opté pour cette composition afin de souligner l'effet du dédoublement de l'image du dédicant et de mise en abyme, ici rendu dans une version plus générique avec des légers décalages entre les deux images. L'image du dédicant sur les pinakes trouvés dans les sanctuaires est générique, attestant parfois son activité professionnelle ${ }^{57}$, son statut social ${ }^{58}$, ou indiquant seulement son nom ou origine avec une inscription ${ }^{59}$. Néanmoins, d'après les sources à notre disposition, il s'agit souvent de l'objet qui parle ${ }^{60}$ et pas de la personne qui l'a déposé sous la protection divine, dont le vœu qui l'accompagnait nous reste inconnu.

Dans d'autres cas, la figure humaine peinte sur le pinax peut être encore plus allusive comme le montre un lécythe à la Villa Giulia ${ }^{61}$. Une jeune femme tenant un kanoun et un porcelet par sa patte arrière ${ }^{62}$ devant un autel allumé s'apprête à offrir aux dieux un sacrifice dans l'objectif de leur demander un service ou de les remercier pour un service déjà rendu, information qui reste non précisée sur les images. L'image peinte sur le pinax rappelle les dessins de base selon lesquels la figure humaine est rendue de façon quasi géométrique avec un point rond pour indiquer la tête et des traits pour son corps et ses extrémités. L'immobilité de la figure sur le pinax redouble l'immobilité de la dédicante dans la scène principale. Néanmoins, aucun élément ne nous permet de reconnaître la personne figurée dans le tableau votif, son genre, ni son identité sociale. Cela ne semble pas être l'objectif du peintre. La présence du tableau votif dans le champ de l'image fonctionne dans la complémentarité avec les autres éléments de la scène faisant partie du système tripartite don, prière, sacrifice selon les formules attestées par les textes littéraires et les inscriptions ${ }^{63}$. Il fait allusion à la dédicante et son geste d'offrande, restant dans le champ de l'image un discret témoignage de cet acte, comme le bucrane, rappelle ailleurs le sacrifice ${ }^{64}$. 
Figure 6

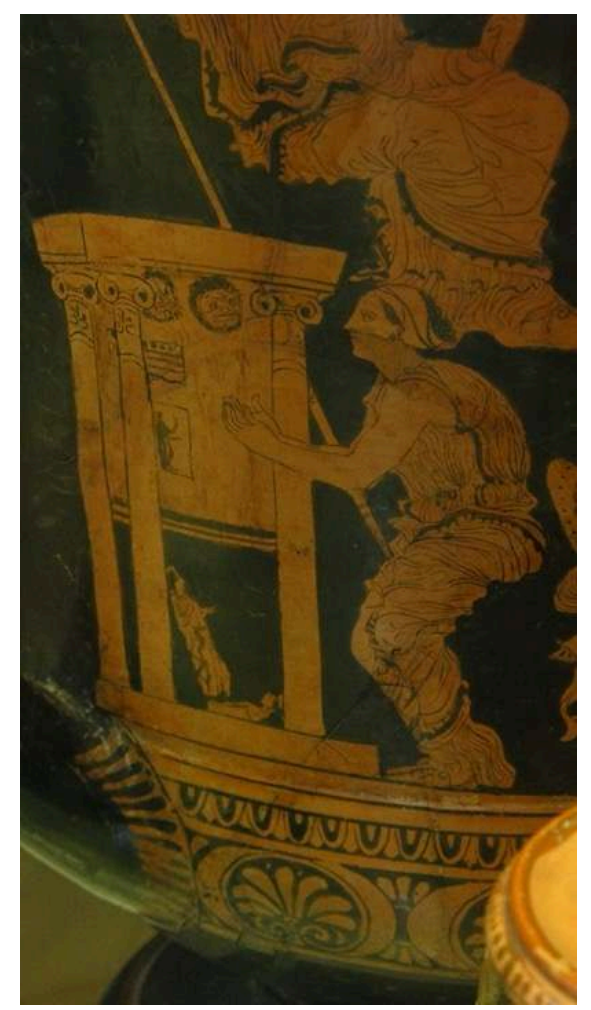

Cratère en cloche lucanien à f.r., Paris, Cabinet des Médailles DR 422, attribué au peintre de Dolon, vers 390.

(c) Vasiliki Zachari

Un autre vase permet d'aborder un cas particulier dans la mesure où est combinée une figure immobile peinte dans le pinax, non pas à une figure humaine, mais à celle d'Athéna (fig. 6) qui s'apprête à faire sa toilette à une fontaine en vue du concours de beauté ${ }^{65}$ avec Aphrodite et Héra. Dans la fontaine au-dessous de deux gargouilles, se trouvent deux pinakes et plus bas des figurines votives. Sur un des tableaux votifs sont inscrits des motifs géométriques, plutôt abstraits, sur lesquels nous reviendrons plus tard, et sur l'autre une figure vêtue d'un vêtement long, probablement féminine, tenant d'une main un objet dans un geste qui rappelle une offrande et élevant l'autre main dans un geste qui pourrait évoquer la prière, selon les exemples qui nous sont familiers dans la céramique attique ${ }^{66}$. La présence de cette image insiste sur l'aspect rituel de l'espace où elle se situe, c'est-à-dire la fontaine, comme le souligne la présence d'autres objets votifs. Cette fonction rituelle des fontaines est reprise abondamment dans les sources littéraires et épigraphiques, notamment pour ce qui concerne les Nymphes ${ }^{67}$, mais elle est très rarement figurée sur les vases. 
Figure 7

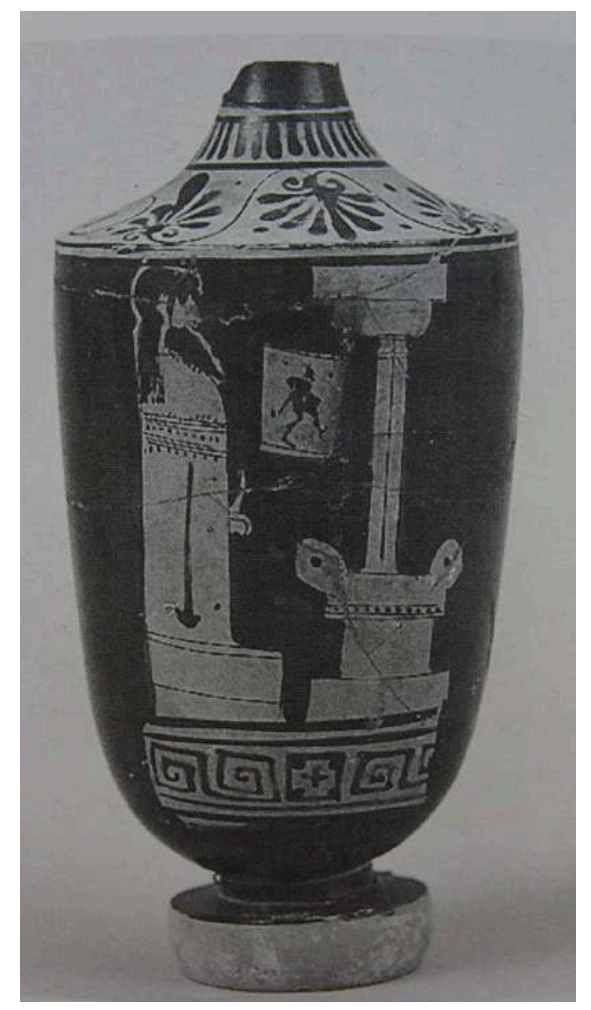

Lécythe à f.r., Palerme, Museo Archeologico Regionale 42354, attribué au peintre de Bowdoin, 470-450 (BAPD 208121).

Rosalba panvinı et Filippo gIUdıce (éds.), Ta Attika. Veder greco a Gela. Ceramiche figurate dall'antica colonia, Rome, L'Erma di Bretschneider, 2003, p. 368, n I 159.

13 Quant aux représentations de figures humaines en mouvement dans les tableaux votifs, sur le cratère de Boston (fig. 3), déjà évoqué préalablement, un jeune homme s'apprête à offrir en sacrifice à Hermès un bouc qu'il maîtrise par les cornes ${ }^{68}$. Sa posture est originale : il chevauche l'animal pour le maîtriser ce qui contraste avec les scènes de sacrifice habituelles où les animaux sont censés aller de leur plein gré au sacrifice ${ }^{69}$. Dans les deux tableaux votifs qui encadrent la statuette d'Hermès, on voit deux personnes s'élancer à grandes enjambées répliquant ainsi le mouvement inscrit dans la scène principale. On ne peut pas déterminer l'identité des deux personnages, il s'agit de figures génériques, d'un homme et d'une femme probablement, qui reprennent et insistent sur l'élan qui porte le jeune homme et le bouc dans la même direction, le dieu Hermès. Cependant, l'effet d'une figure en mouvement n'est pas toujours le même. Sur un lécythe à Palerme (fig. 7) la scène se construit autour des éléments architecturaux créant un espace immobile. Sur le pinax figuré dans le champ de l'image nous n'avons pas affaire à une mise en abyme avec le dédoublement de l'image de l'hermès. En revanche, le personnage représenté à l'intérieur du tableau votif est pris dans un mouvement de tout son corps, seul élément apportant une animation à la scène par ailleurs totalement figée. Ces deux cas attestent les options possibles de jeux d'images soit avec un effet d'accentuation soit de contraste entre la scène principale et l'image dans le pinax.

Dans un premier temps on peut constater la rareté des images divines présentes sur les pinakes à l'exception de la statue d'Hermès au contraire très fréquente. Ensuite, les 
figures humaines représentées sont génériques et font souvent allusion au protagoniste de la scène. Les peintres ne reproduisent pas la réalité rituelle, mais ils s'appliquent à mettre en valeur majoritairement des jeux d'images en recourant à la mise en abyme, aux images allusives et réflexives en s'appuyant sur les éléments de la scène principale aboutissant ainsi à des créations sophistiquées et originales.

\section{Animaux et êtres hybrides}

Figure 8

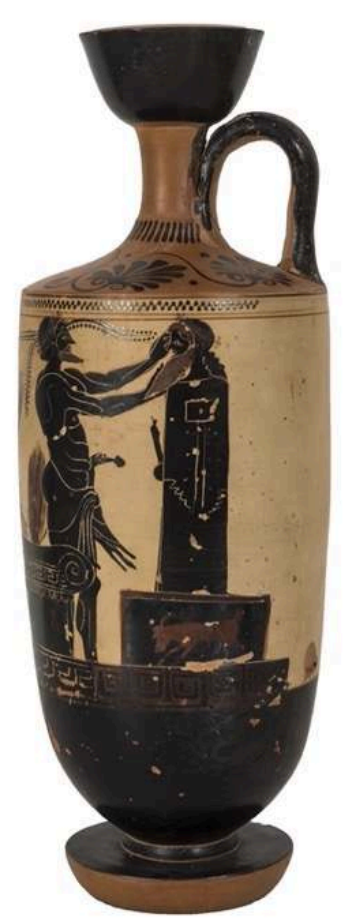

Lécythe à f.n., Pirée Musée Archéologique 7339 attribué au peintre de Thésée, vers 490.

(c) Ministère de la culture et d'athlétisme. Ephorie de l'Attique de l'ouest, Pirée et îles.

Dans cette catégorie, les exemples sont plus limités par rapport aux autres groupes d'images, ils méritent, néanmoins, notre attention pour mieux comprendre l'effet que ces détails discrets suscitent chez les spectateurs et le sens qu'ils apportent à la scène. Sur les vases analysés jusqu'à présent, les pinakes figurent dans le champ de l'image et en hauteur, souvent dans le prolongement de l'autel, comme les bucranes. Or, sur un lécythe attribué au peintre de Thésée (fig. 8), montrant une procession sacrificielle arrivant devant un autel allumé et un hermès, l'image d'un bouc - reconnaissable grâce à ses courtes pattes, son corps oblong, sa queue et ses longues cornes - est peinte en couleur rougeâtre ${ }^{70}$ à côté de la partie basse de la statue du dieu Hermès. Est-ce qu'il s'agit d'un pinax placé à proximité de la statue ou de la base du pilier décorée ? Effectivement, certaines sources ${ }^{71}$ mentionnent la pratique des fidèles plaçant leur offrande au plus près de la protection divine, l'accrochant même parfois sur la statue si possible, à tel point que des restrictions ont été imposées dans les sanctuaires afin de préserver l'état des statues divines et des bâtiments, selon les témoignages de certaines 
inscriptions $^{72}$. En ce qui concerne la représentation de cette pratique sur les vases, elle ne semble pas être courante dans le répertoire connu; parfois des couronnes et des branchages sont accrochées aux statues divines et aux autels ${ }^{73}$. En revanche, les peintres de vases mettent en avant les offrandes maintenues ${ }^{74}$, celles déposées sur l'autel ${ }^{75}$ et enfin celles figurées dans le champ de l'image ${ }^{76}$. Ainsi, la dernière proposition nous semble la plus plausible, à savoir qu'il s'agit bien de la base de la statue peinte, si on prend également en considération deux conditions : d'abord, les statues mêmes dont la base est parfois ornée d'images - comme la célèbre statue de Némésis provenant de Rhamnonte ${ }^{77}$ - et ensuite, l'exécution des piliers hermaïques sous le pinceau du peintre de Thésée. En effet, les hermès dans ses créations sont dotés d'une base si haute qu'elle devient parfois support graphique, orné de motifs linéaires gravés $^{78}$. Néanmoins, il n'y a guère de doute quant à l'identification d'un pinax avec un bouc figuré dans le champ de l'image d'un skyphos à Salerne ${ }^{79}$ attribué au même peintre et le situant dans une scène d'atelier du forgeron. Dans les deux vases, le peintre de Thésée aboutit à des créations intéressantes et variées avec la figuration du bouc, un de ses sujets de prédilection et marque de fabrication de ses vases ${ }^{80}$, figurant souvent sous les anses des skyphoi ${ }^{81}$ et comme unique sujet de représentation sur un plat $^{82}$.

Figure 8bis
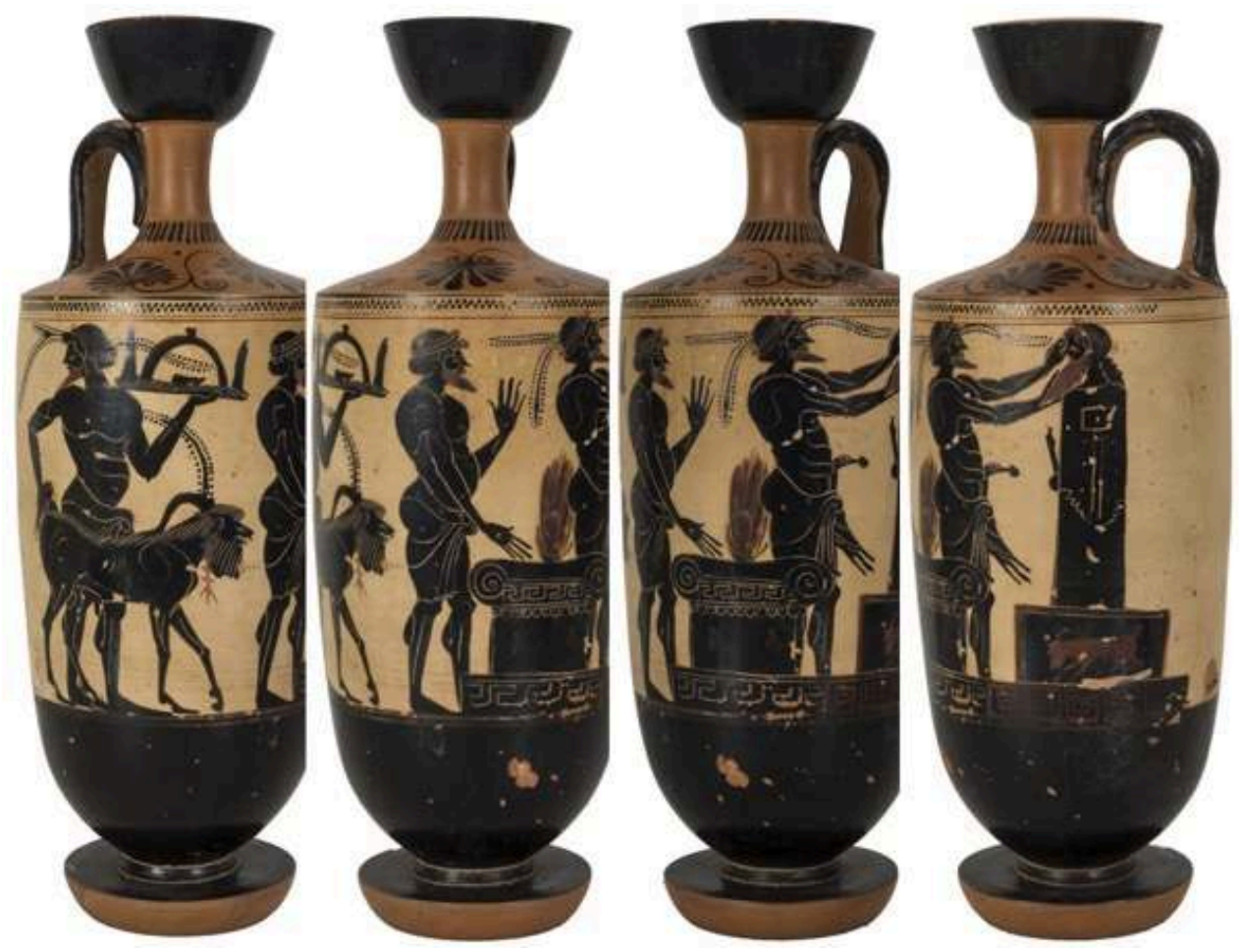

Image en déroulée

Les jeux d'images dans les deux cas sont davantage soulignés par le support de l'image, à savoir la forme du vase, ainsi que la technique d'exécution qui fait exception par rapport aux autres exemples dans le corpus des images avec pinakes. Dans le lécythe du Pirée, l'emplacement excentrique de l'image du bouc crée une ambiguïté graphique entre pinax posé et base de la statue peinte - mais aussi une surprise visuelle ${ }^{83}$ avec une mise en abyme que le spectateur n'arrive pas à apercevoir instantanément au moment 
où il regarde le bouc peint sur la base de l'hermès. En effet, le lécythe, une forme de vase oblongue, ne permet pas la visualisation simultanée de l'ensemble de la scène peinte sur son corps. En tournant le vase, le spectateur découvre progressivement les autres éléments de la scène, l'autel allumé, les participants, trois hommes barbus, et enfin l'animal qu'ils offrent en sacrifice au dieu, plus précisément un bouc. Ainsi, les images de l'animal, avec sa présence physique et sa représentation iconique, l'une faisant face à l'autre comme par un effet de miroir, encadrent la scène, rappelant à chaque fois le contexte sacrificiel ${ }^{84}$. Le skyphos, de l'autre, un vase de forme ouverte, permet que la scène de chaque surface soit vue dans son intégralité sans manipulation particulière. Dans le cas de Salerne, on retrouve l'image du bouc, dans un contexte non rituel, sur un pinax situé de façon standard en hauteur à proximité du four du forgeron $^{85}$. Le jeu d'image ici s'appuie sur l'aspect chromatique, la couleur rouge utilisée pour le bouc, ainsi que pour le pais, le jeune homme accroupi au bas du four. Ce rappel de couleur inscrit un dialogue entre les deux éléments et pourrait suggérer une assimilation du jeune homme à un statut d'objet ou d'animal. Les vases du corpus de pinakes sont quasiment tous à figures rouges, comme les images avec bucrane. Les créations du peintre de Thésée mentionnées ici font exception dans la série pour ce qui concerne leur technique d'exécution, et par conséquent, celle de l'image peinte dans l'image avec l'inversion de couleurs: sujet rouge sur fond noir. Le skyphos à Salerne daté au début $\mathrm{du} \mathrm{v}^{\mathrm{e}} \mathrm{s}$. constitue la première attestation de pinakes sur les vases à figures noires qui nous permet de remonter dans le temps l'apparition du motif du tableau peint dans le champ de l'image qui deviendra courant sur les vases à figures rouges dès 480 . 
Figure 9

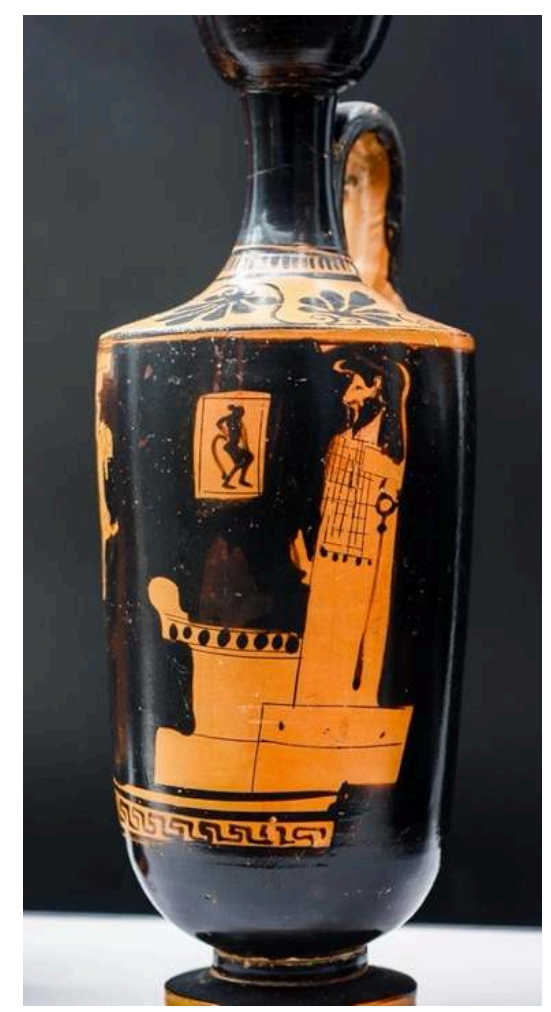

Lécythe à f.r., Karlsruhe, Badisches Landesmuseum 85.1, attribué au peintre de Bowdoin, 480-470 (BAPD 208122).

(C) Wikimedia Commons

Pour ce qui concerne les êtres hybrides, nous disposons de deux cas, le satyre et la sirène, que nous abordons au travers de deux lécythes appartenant au même groupe de peintres et datés de la même époque. Sur un lécythe à Karlsruhe (fig. 9) nous nous retrouvons dans le même registre avec un autel et un pilier hermaïque, un pinax suspendu dans le prolongement de l'autel et une colonne dorique d'où pend un lièvre. Les nouveaux éléments dans cette composition sont l'image peinte dans le tableau votif et le gibier. Plus précisément, dans le pinax posé face au pilier hermaïque, on distingue grâce à sa queue un satyre accroupi, qui fait avec sa présence exception au jeu de mise en abyme habituelle avec le dédoublement de l'image divine. Le peintre de Bowdoin semble avoir associé ces motifs de façon à mettre en avant un autre jeu d'image moins explicite. Pour mieux le saisir, il faudra d'abord repérer ce qu'il peut y avoir en commun entre le satyre et l'hermès. L'élément graphique partagé entre les deux qui nous semble parlant est leur phallus. Attribut distinctif et symbolique du pilier hermaïque, son phallus en érection capte l'œil du spectateur et entraîne parfois des gestes intimes de la part des fidèles comme l'attestent certains vases ${ }^{86}$; il peut être considéré comme symbole de fécondité ou de porte-bonheur ${ }^{87}$. Quant aux satyres, créations de l'imaginaire des peintres, ils sont représentés en érection quasi permanente et démesurément ithyphalliques, un effet visuel qui cherche à provoquer le rire surtout dans des scènes où leur sexe sert de cinquième membre ${ }^{88}$. Même si cet aspect n'est pas mis explicitement en avant sur l'image du satyre sur le pinax, dont on ne distingue pas le sexe ${ }^{89}$, cela ne surprend pas pour autant, si on prend en considération le contexte de la scène. En effet, quand les satyres entrent dans l'espace de la cité et plus précisément dans les images rituelles et des actes religieux, ils sont 
souvent dessinés "sages", sans érection" ${ }^{90}$. Par cette juxtaposition, le peintre de Bowdoin cherche probablement à créer une association moins graphique que mentale.

Figure 10

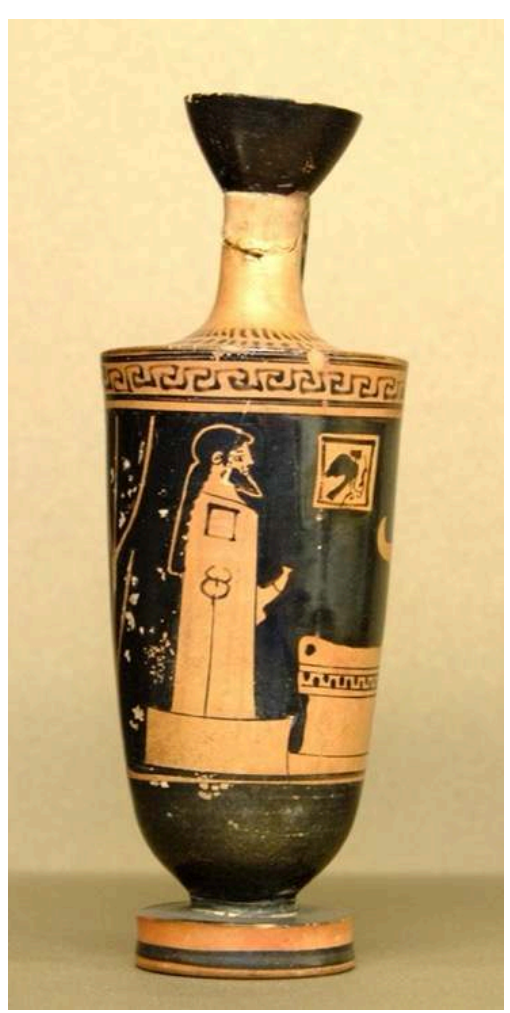

Lécythe à f.r., Paris, Musée du Louvre CA 2935, 470-450 (BAPD 9026895).

(C) Snappy Goat

Dans le deuxième lécythe au Louvre nous nous trouvons face à une énigme : comment interpréter la présence d'une sirène sur le pinax faisant face à hermès (fig. 10) ? La réponse est peut-être à chercher également dans les éléments que ces deux figures possèdent en commun. À première vue, il n'existe pas d'élément graphique identique entre les deux. Les premières attestations iconographiques de cet être mixte à l'apparence d'oiseau anthropocéphale que l'on appelle sirène datent du viII ${ }^{\mathrm{e}} \mathrm{s}$. dans la zone de la Méditerranée orientalisante ${ }^{91}$ et sa figuration devient fréquente sur les vases de la période archaïque et classique dans des contextes variés ${ }^{92}$. Qu'il s'agisse de scènes de course ou de départ de guerrier, de scènes funéraires ou mythologiques parmi d'autres, la présence de la sirène a souvent été interprétée comme symbole de protection et de bienveillance ${ }^{93}$, ou chthonien ${ }^{94}$, ainsi que comme une créature de l'entre-deux ${ }^{95}$, un être liminaire ${ }^{96}$. Une série de vases provenant du sanctuaire de la Nymphe au pied de l'Acropole d'Athènes permet de souligner davantage la dernière proposition. Il s'agit de vases nuptiaux, dont l'utilisation et l'iconographie sont étroitement liées, où la présence de la sirène dans des endroits différents, sous l'anse du vase ou dans la scène principale, sert à marquer la transition et le changement de statut de la jeune fille en épouse ${ }^{97}$. Cette notion de passage d'un état à un autre, un aspect qui n'est pas iconique à proprement parler, mais plutôt conceptuel, rapproche la sirène et le pilier hermaïque en termes de rapports d'homologie. En effet, l'image du dieu Hermès sous toutes les figurations possibles «représente le mouvement, le 
passage, le changement d'état, des transitions, les contacts entre éléments étrangers ${ }^{98}$ ». Dans cette perspective, nous arrivons à mieux comprendre la présence de la sirène sur le pinax du lécythe au Louvre comme un être qui sert éventuellement à marquer le passage d'un vœu - matérialisé par la présence du tableau votif ${ }^{99}$ - à sa réalisation ; une transition entre l'univers des fidèles et celui des divinités.

19 À l'opposé de l'image du pilier hermaïque et du satyre, celles du bouc et de la sirène sont attestées sur quelques pinakes. Ces exemples ne sont pas assez nombreux pour nous permettre une étude détaillée. Leur présence dans les realia ne signifie pas une reproduction fidèle sur les vases. Encore une fois, les peintres s'inspirent de leurs créations pour faire des associations mentales, plus faciles à déchiffrer pour le spectateur de l'Antiquité que pour nous.

\section{Motifs abstrait et pinakes vides}

Jusqu'à présent, nous avons regardé de près les images des pinakes ayant comme sujet des thèmes figuratifs précis, dont certains en lien avec le contexte rituel de la scène. Nous passons enfin à une série de pinakes particuliers qui comporte des images composées d'éléments géométriques, voire de pinakes dépourvus de la moindre figuration. Même si ces sujets nous semblent inattendus, leur présence n'est pas du tout négligeable dans le corpus et ils méritent un regard plus attentif afin de mieux comprendre les intentions des peintres, puisqu'ils ouvrent de nouvelles perspectives dans l'interprétation de tableaux votifs peints sur les vases et proposent quelques réponses intéressantes sur le sujet de l'image dans l'image. 
Figure 11

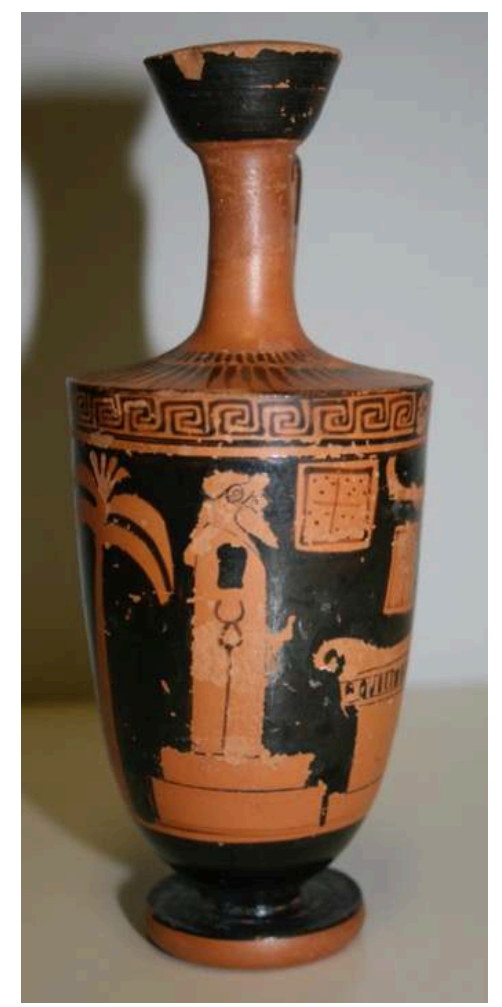

Lécythe à f.r., Sydney, Université Nicholson Museum 51.14 attribué au peintre d'Icare, 480-470 (BAPD 208347).

(c) François Lissarrague

En ce qui concerne les pinakes comportant des éléments graphiques élémentaires, à savoir des lignes et des points, nous allons les aborder à partir de deux exemples représentatifs. Sur un lécythe à Sydney (fig. 11) nous trouvons les éléments habituels de l'espace sacré : un palmier, un hermès et un autel avec une paire de cornes et deux tableaux votifs placés dans le prolongement de l'autel avec un léger décalage pour l'un des pinakes. À l'intérieur on distingue une croix avec des traits très fins qui partagent l'espace en quatre parties dans lesquelles se trouvent disséminés des points éparpillés ${ }^{100}$. Le peintre a pris le soin d'encadrer le contenu de deux pinakes soulignant ainsi sa volonté de leur attribuer une valeur notable, comme pour les autres éléments figurés. Comment pourrions-nous saisir la présence de la croix entourée de points à l'intérieur des tableaux votifs? Le motif de la croix est récurrent sur les vases aux zones décoratives qui encadrent la scène principale, notamment dans les médaillons de coupes, et il se trouve en plusieurs variations en alternance avec des méandres et d'autres ornements ${ }^{101}$. En l'isolant et en le mettant dans le pinax, le peintre a voulu éventuellement faire un clin d'œil à ses autres créations artistiques et susciter avec ce jeu de l'image dans l'image un moyen pour proclamer l'art du pinceau et les productions de l'atelier ${ }^{102}$.

Sur le cratère lucanien au Cabinet des Médailles (fig. 6), le pinax vertical avec l'image du dédicant est placé au-dessous d'un pinax horizontal - figurant en deux parties à cause de la colonne de la fontaine peinte devant lui - composé d'une série de points peints dans la partie supérieure du tableau et de trois lignes horizontales parallèles audessous des points. Cet ensemble dans le tableau votif ne correspond pas aux motifs 
ornementaux connus dans la céramique et sa présence semble étrange dans une composition aussi élaborée que celle de la scène dédiée à la préparation de déesses pour le jugement de Pâris où ce pinax est situé. Néanmoins, la volonté du peintre de rendre minutieusement plusieurs détails dans la scène, dont les gargouilles, le reflet d'Aphrodite au miroir, le gorgoneion ${ }^{103}$ sur le bouclier d'Athéna, ne nous permet pas d'avancer l'hypothèse qu'il s'agit d'un simple remplissage du tableau votif avec des motifs quasi abstraits à nos yeux. Au contraire, cela semble correspondre à un choix réfléchi, comme le confirment la figuration de ce motif dans plusieurs exemplaires : sur un autre vase à Naples ${ }^{104}$, ainsi que sur quelques tableaux votifs provenant du sanctuaire de Déméter à Éleusis. En effet, à notre surprise, quelques pinakes sont recouverts des motifs linéaires et des quadrillages, ainsi que d'autres motifs en $\mathrm{x}$, triangle, filet, rosette ${ }^{105}$. La question se pose bien évidemment de la signification de ces motifs abstraits sur les tableaux votifs à laquelle nous tenterons de répondre après avoir étudié la dernière série, celle de pinakes dépourvus de contenu figuratif. Que se passe-t-il quand une « image - objet » reste vide? Quelle place tient-il dans l'image et quel est son apport à la scène principale ? Enfin, est-ce l'image qui compte ou le geste d'offrir un pinax à une divinité ?

Sur les vases que nous allons analyser à présent, les pinakes qui figurent dans le champ de l'image sont dépourvus de la moindre représentation. Ce choix des peintres ne nous semble pas être un hasard ou une exception dans le corpus de pinakes et montre que les peintres attiques n'ont pas peur du vide, au sens littéral du terme.

Figure 12

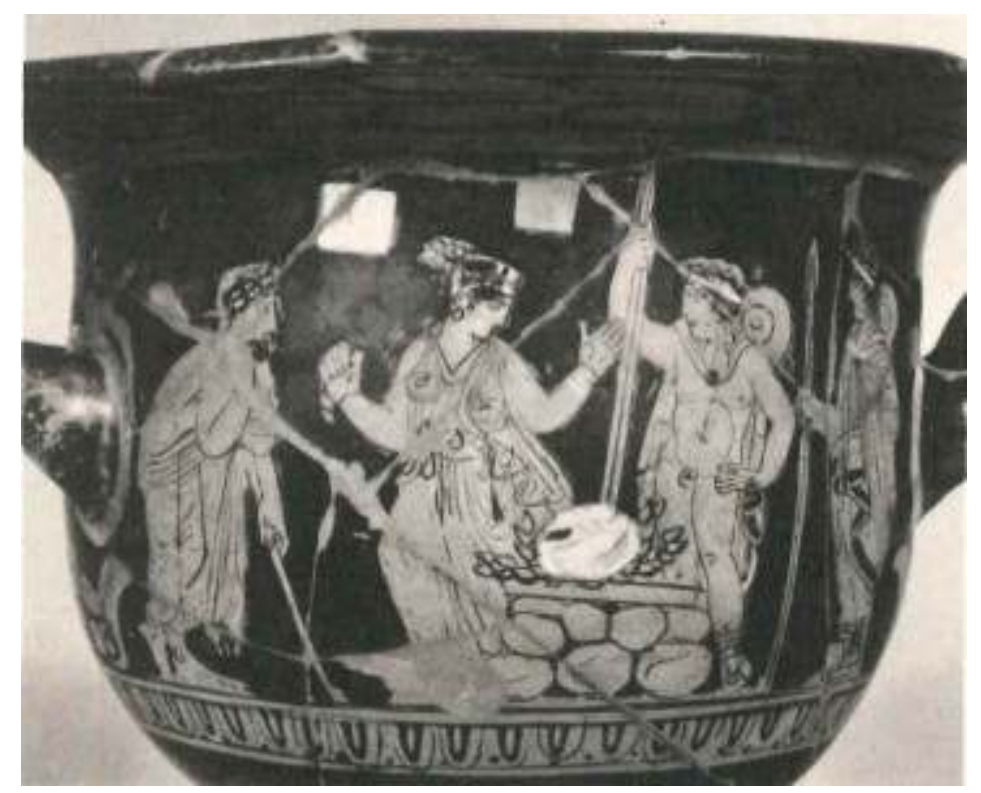

Cratère en cloche à f.r., Vienne, Kunsthistorisches Museum 2000, attribué au peintre de Nikias, vers 400 (BAPD 217478).

CVA Vienne Kunsthistorisches Museum 3, pl. 122.

Un cratère de Vienne (fig. 12) permet de mieux comprendre le rapport entre le vide et le plein mis en avant par l'effet de l'image dans l'image et comment ils « s'engendrent réciproquement ${ }^{106} »$. Le peintre de Nikias a adopté un sujet exceptionnel dans le répertoire de la céramique attique (et italiote) pour décorer ce cratère : il s'agit de la naissance d'Hélène à partir d'un œuf posé sur un autel ${ }^{107}$. De part et d'autre de l'autel 
figure la famille d'Hélène, ses frères, les Dioscures à droite et ses parents mortels Tyndare et Léda à gauche. Dans le champ de l'image sont peints deux carrés, deux pinakes vides, sans représentation quelconque. Quel pourrait être le rôle de ces pinakes dans une scène sans contexte rituel explicite et pourquoi le Peintre de Nikias a-t-il choisi de ne rien indiquer à l'intérieur de ces surfaces susceptibles de recevoir une image? Du point de vue graphique, les deux tableaux votifs encadrent Léda, lui conférant ainsi un rôle primordial dans la scène, et concentrent l'attention du spectateur sur son geste d'étonnement face à la naissance surprenante. Devant cette scène puissante très évocatrice le peintre a décidé de ne pas entrer dans le détail dans les pinakes qui pourrait diminuer son intensité. Du point de vue sémiologique, la présence de pinakes indique l'espace rituel ${ }^{108}$, mais devient aussi, comme le bucrane, signe de temporalité indiquant la trace d'un acte de piété humaine, celui de l'offrande.

\section{Figure 13}

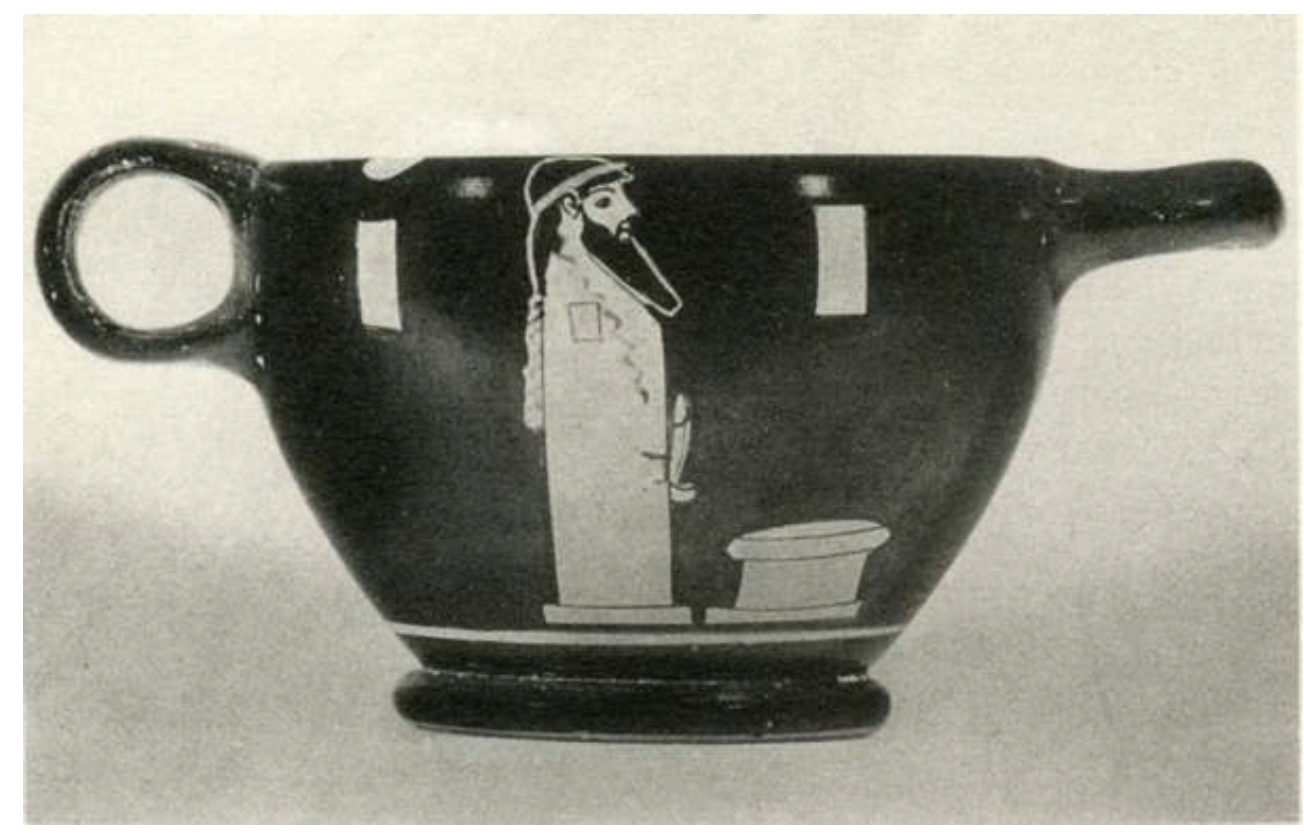

Skyphos à f.r., Berlin Antikensammlung F 2594, attribué au peintre de Triptolème, vers 450 (BAPD 203896).

CVA Berlin Antikensammlung 3, pl. 141. 


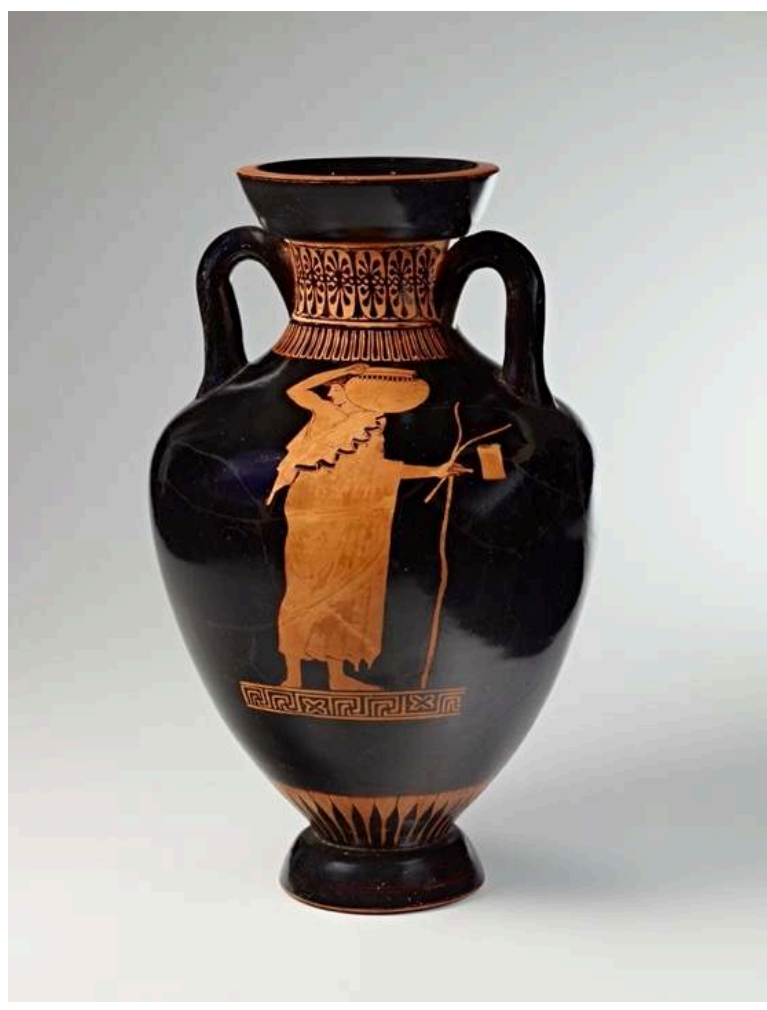

Amphore panathénaïque à f.r., Stanford, Cantor Center for the Visual Arts 1970.11, attribué au peintre d'Eucharidès, vers 480 (BAPD 9761).

(C) Iris \& B. Gerald Cantor Center for Visual Arts at Stanford University; Hazel D. Hansen Fund. peintre de Triptolème (fig. 13), un vase qui fait partie de la série des pinakes à l'espace vide, c'est-à-dire dépourvu de figuration humaine ${ }^{109}$. Sur les deux faces du vase la même composition se répète, à savoir un autel avec pilier hermaïque encadrés par deux pinakes vides. Il s'agit d'un jeu de dédoublement et de répétitions scandant l'espace, ainsi que d'une insistance sur le vide dans la scène principale comme dans les pinakes. Nous comprenons, ainsi, leur fonctionnalité comme indice de spatialité marqueur du sacré, ainsi que de temporalité, un signe du geste de l'offrande subtilement évoquée dans le champ de l'image. Dans ce contexte le vide de la scène principale souligne l'absence physique de l'homme, mais pas de son acte évoqué avec les deux tableaux votifs. Enfin, le vide, l'éclipse de figuration, dans chaque pinax, le fait apparaître non seulement comme une image suffisante, juste un carré rouge dans le champ de l'image, mais aussi comme une image efficace qui souligne ainsi la puissance du geste dédicatoire, tel qu'on le voit rendu de façon explicite sur d'autres vases (fig. 14).

Pour conclure, les sanctuaires étaient élaborés comme des "communautés conceptuelles d'images » où les offrandes votives avec leurs images étaient en même temps acceptées par les divinités et reconnues par les hommes ${ }^{110}$. Pour ce qui concerne la représentation d'images votives sur les vases, nous avons montré à partir de l'exemple des pinakes qu'il s'agit d'un objet conceptuel d'images ayant des fonctions structurantes dans la scène principale comme indicateur de l'espace sacré et créant des jeux d'image avec les mises en miroir et en abyme. Lors de cette analyse nous nous sommes arrêtée à plusieurs reprises sur des scènes associant autel et pilier hermaïque, 
éléments architecturaux qui sont eux-mêmes des anathemata ${ }^{111}$, comme les pinakes. Ces créations sont figurées sur des vases souvent destinés à être offerts comme offrande soit dans le sanctuaire, soit dans la tombe ${ }^{112}$. Nous ne pouvons pas savoir à quel moment le peintre pensait à l'ensemble de sa composition. Il est plausible qu'il exécutait le dessin du tableau votif après avoir peint la scène principale. Le temps de changer de pinceaux et de s'approcher de la surface du vase, il avait un large éventail d'options parmi lesquelles choisir, selon l'effet qu'il souhaitait créer: une mise en abyme ou une image réflexive - dans tous les cas, un jeu d'auto-référentialité avec ses créations et les pratiques cultuelles qui lui étaient contemporaines.

\section{NOTES}

1. Pour une présentation des variations possibles, voir l'introduction de Giulia Puma: https:// journals.openedition.org/imagesrevues/8727.

2. François lisSARRAGUE, «Le temps des boucliers ", dans Giovanni CARERI, François LISSARRAGUE, Jean-Claude SCHMITT et Carlo SEVERI (éds.), Traditions et temporalités des images, Paris, EHESS, 2009, p. 21-31, version en ligne dans Images Re-vues, H.S. 1, 2008 (http://journals.openedition.org/ imagesrevues/850).

3. Alain SCHNAPP, «Why did the Greeks need Images?», dans Jette CHRISTIANSEN et Torben MELANDER, Ancient Greek and Related Pottery: Proceedings of the $3^{\text {rd }}$ Symposium, Copenhaguen, August 31 September 4 1987, Copenhague, Thorvaldsens Museum, 1988, p. 569.

4. Jérôme BASCHET, L'iconographie médiévale, Paris, Gallimard « folio histoire », 2008, p. 45.

5. Georges DIDI-HUBERMAN, «Imitation, représentation, fonction. Remarques sur un mythe épistémologique » dans L'image. Cahiers du Léopard d'Or, 5, 1996, p. 59.

6. HOMÈRE, Odyssée 1.141 et 16.49 .

7. HOMÈRE, Iliade 6.169.

8. Pour cette raison les lexicographes font l'association avec le terme sanis.

9. Sur les termes de pinakes: Pierre CHANTRAINE, Dictionnaire étymologique de la langue grecque. Histoire des mots, Paris, éditions Klincksieck, 1968, p. 903 ; Marie-Christine HELLMANN, Recherches sur le vocabulaire de l'architecture grecque, d'après les inscriptions de Délos, BEFAR 278, Athènes Paris, École française d'Athènes - de Boccard, 1992, p. 88, 91-93 ; Kyriaki KAROGLOU, Attic Pinakes: Votive images in clay, Oxford, Archaeopress, 2010, p. 5-10.

10. François LISSARRAGUE, "Graphein: écrire et dessiner", dans Christiane BRoN et Effy KASSAPOGLOU (éd.), L'image en jeu de l'antiquité à P. Klee, Yens-sur-Morges, Cabédita, 1992, p. 189-203.

11. K. KAROGLOU, Attic Pinakes, op. cit., p. 5.

12. Sur les portraits peints : Vasiliki BOURA, La peinture de portraits à l'époque hellénistique et romaine, thèse soutenue en 2014 sous la direction d'Agnès Rouveret.

13. Sur les tableaux votifs: K. KAROGLOU, Attic Pinakes, op. cit.; John BOARDMAN et al. «Dedications. Greek dedications ", dans ThesCRA I 2.d, Los Angeles, J. Paul Getty Museum, 2004, p. 293-296; Anneliese KOSSATZ-DEISSMANN, «Représentations of cult places », dans ThesCRA IV 1.b, Los Angeles, J. Paul Getty Museum, 2005, p. 402-408.

14. Pour les objets votifs en terre cuite: Gina SALAPATA, "Greek votive plaques. Manufacture, display, disposal », Bulletin Antieke Beschaving 77, 2002, p. 19-42 
15. Par exemple ceux provenant de la grotte de Pitsa en Corinthe.

16. Frederick nAEREBout, «Baked Prayers. The Penteskouphia Plaques in the Context of Ritual Depositing" présenté lors du colloque "Offerings in Clay: Votive Plaques in Ancient Greek Religion” lors de la 106e rencontre annuelle de l'AIA à Boston les 6-9 janvier 2005.

17. La présence des dédicaces peintes avant la cuisson du vase est assez rare. C'est essentiellement après qu'elles ont été ajoutées, surtout par incision, ou sinon peintes en couleur violette ou blanche. K. KAROGLOU, Attic Pinakes, op. cit.

18. Claudia WAGNER, "The Potters and Athena. Dedications on the Athenian Acropolis ", dans Gocha TSETSKHLADZE et Anthony SNODGRASS, Periplous. Papers on classical Art and Archaeology presented to Sir John Boardman, Londres, Thames \& Hudson, 2000, p. 383-387.

19. Dans l'archive Beazley 31 vases sont répertoriés sous le terme «PINAX » et 11 sous le terme «PINAKES », mais parfois quelques cas ne correspondent pas à ce motif. Dans notre catalogue nous comptons 49 vases dont 42 attiques. Le corpus est susceptible de croître avec la découverte fortuite d'autres vases italiotes. Il y a quelques exceptions au niveau de la technique que nous allons aborder par la suite.

20. Verity PLATT et Michael SQUIRE (éds.), The Frame in classical Art: a cultural History, Cambridge, Cambridge University Press, 2017.

21. Participants aux fêtes en honneur de Dionysos, au banquet.

22. Coupe à f.r., Oxford, Ashmolean Museum 1973.1 attribuée au peintre de la Villa Giulia, vers 450 (BAPD 802).

23. Dans la publication du fragment il est également proposé d'y voir Zeus (CVA p. 41, pl. 29.2). Le manque de parallèles ne nous permet pas de confirmer cette suggestion.

24. Lécythe à f.r., Tübingen, Eberhard-Karls-Universität, Archäologisches Institut 5606, comparer avec peintre d'Icare, vers 450 (BAPD 16864).

25. Cratère en calice à f.r., Athènes, Musée Archéologique 12491, 410-400 (BAPD 46072).

26. En général une seule figure est représentée à l'intérieur d'un tableau, mais il peut en avoir plusieurs : cratère à colonnettes à f.r., Naples, Museo Archeologico Nazionale 81295 attribué au peintre du Verger, 470-460 (BAPD 205886).

27. Skyphos à f.r., Paris, Cabinet des Médailles 839, attribué au peintre de Triptolème, vers 470 (BAPD 203889).

28. Chous à f.r., Paris Musée du Louvre L 64 à la manière du peintre de Meidias, vers 410 (BAPD 15906).

29. Ampore panathénaïque à f.r., Bâle, marché des antiquités, attribué au peintre d'Eucharidès, vers 480 (BAPD 9761).

30. Fragment de pélikè à f.r., Florence, Museo Archologico Etrusco 14B26, à la manière du peintre de Hasselmann, 440-430 (BAPD 215094).

31. Sur quelques vases, il peut $y$ avoir de deux à sept pinakes: skyphos à f.r., Berlin, Antikensammlung F 2594, attribué au peintre de Triptolème, vers 450 (BAPD 203896); skyphos italiote à f.r. à Gela, Museo Nazionale, 350-300.

32. Sur la question de la spatialité des pinakes: Vasiliki ZACHARI, «Tableaux votifs et spatialité dans la céramique attique $\left(\mathrm{vI}^{\mathrm{e}}\right.$ et $\mathrm{v}^{\mathrm{e}} \mathrm{s}$.)», Techniques \& Culture 70 "Matérialiser les désirs. Techniques votives », 2018, p. 58-79 ; DOI : https://doi.org/10.4000/tc.9478.

33. Les plaques en relief, comme les figurines en terre cuite, étaient fabriquées par les coroplastes selon G. SALAPATA, «Greek votive plaques... », op. cit., p. 21.

34. Jean-Louis DURAND et François LISSARRAGUE, «Un lieu d'image? L'espace du loutérion», Hephaistos 2, 1980, p. 89-106; Jean-Louis DURAND, "L'Hermès multiple ", dans C. BRon et E. KASSAPOGLOU (éd.), L'image en jeu, op. cit., p. 25-34 ; Vasiliki ZACHARI, « Image de l'espace ou espace de l'image?", Cahiers «Mondes anciens» [En ligne], 4 | 2013, mis en ligne le 06 juillet 2013. URL : 
http://journals.openedition.org/mondesanciens/1087 ; DOI : https://doi.org/10.4000/ mondesanciens.1087.

35. Sur les piliers hermaïques vêtus dans l'art romain voir Stéphanie WYLER, "L'habit fait-il le dieu? Gestes et parures autour des hermes rustiques dans l'art romain " dans Florence GHERCHANOC et Valérie HUET (éd.), Performances corporelles et vestimentaires en Grèce et à Rome: le rituel en question (à paraître).

36. Evelyn HARRISON, The Athenian Agora vol. XI. Archaic and archaistic sculpture, Princeton, American School of Classical Studies at Athens, 1965, p. 141.

37. Lécythe à f.r., Mississippi, University of Mississippi Museum 1977.003.0199, attribué au peintre d'Icare, vers 470 (BAPD 208344).

38. Pélikè à f.r., Boston, Museum of Fine Arts 13.100, rappelle le peintre de Hasselmann, 440-430 (BAPD 215101).

39. On trouve une exception sur le skyphos du Cabinet des Médailles (note 26) où deux pinakes identiques encadrent le pilier hermaïque et l'un des deux ne suit pas cet effet. Sur le cratère apulien à Bruxelles A 725 le pinax porte l'image d'un pilier hermaïque vu de face et encadré par deux figures.

40. Gérard SIEBERT, «Une image dans l'image. Le pilier hermaïque dans la peinture de vases grecque », dans Françoise DUNAND, Jean-Michel SPIESER et Jean WIRTH (éd.), L'image et la reproduction du sacré. Actes du colloque de Strasbourg (20-21 janvier 1988), Paris, Méridiens Klincksieck, 1991, p. 107.

41. Ces objets sont étudiés dans le cadre de la thèse de Vasiliki zACHARI, Autour de l'autel. Spatialité, temporalité et gestualité dans la céramique attique $d u v^{e}$ et $d u v^{e}$ s. av. J.-C. (EHESS, Paris, sous la direction de François Lissarrague).

42. Il s'agit notamment des sujets mythologiques qui sont favorables à cette association. Hélène COLLARD, "Montrer l'invisible. Les dieux et leurs statues dans la céramique grecque », dans Philippe BORgEAUd et Doralice faBIANo (éds.), Perception et construction du divin dans l'Antiquité, Genève, Droz, 2013, p. 61-86.

43. Sur les images du sacrifice : Folkert VAN STRATEN, Hierà kalá: Images of animal Sacrifice in Archaic and Classical Greece, Leiden \& New-York, Brill, 1995 ; Jörg GEBAUER, Pompe und Thysia. Attische Tieropferdarstellungen auf schwarz- und rotfigurigen Vasen, Münster, UGARIT-Verlag, 2002.

44. Sur les jeux de visibilité-invisibilité : Lorenz E. BAUMER, « Entre dieux et mortels - le contact visuel sur les reliefs votifs grecs classiques», Pallas, $92 \mid 2013$, p. 43-54; mis en ligne le 29 novembre 2013. URL : http://journals.openedition.org/pallas/95 ; DOI : https://doi.org/10.4000/ pallas.95. Hélène COLLARD, Montrer l'invisible. Rituel et présentification du divin dans l'imagerie attique (Kernos supplément 30), Liège Presses Universitaires de Liège, 2016.

45. Cette interprétation est basée sur l'analyse du relief votif à Athènes, Musée National 1977, provenant du sanctuaire d'Asclépios par Verity PLATT, « Framing epiphany in art and text » dans ead., Facing the Gods. Epiphany and Representation in Graeco-Roman Art, Literature and Religion, Cambridge, Cambridge University Press, 2011, p. 31-50.

46. Les plus anciennes attestations se trouvent dans les plaques votives provenant du sanctuaire

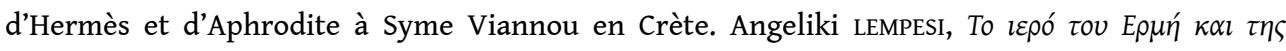

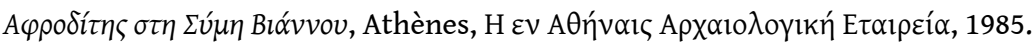

47. Avec Athéna sur le pinax à Athènes, Musée National coll. Acr. 2547 ; dans une assemblée des dieux, Athènes, Musée National coll. Acr. 1047.

48. Francis PROST, "Conclusions", dans Sylvia ESTIENNE, Valérie hUET, François LISSARRAGUe et Francis PROST, Figures de dieux. Construire le divin en images, Rennes, Presses Universitaires de Rennes, 2014, p. 364.

49. L'identification des objets n'est pas aisée : il s'agit probablement des morceaux de viandes ou d'étoffes selon le catalogue du musée. En effet, dans la série dont ce vase fait partie on voit des 
morceaux de viande suspendus à la table et des étoffes placés sur les klinai. Ici, la forme des objets posés sur la table est ambiguë et le rendu avec des incisions et du rehaut blanc qui n'est pas bien préservé ne nous permet pas de bien les distinguer.

50. De même pour l'inscription PAIS qui court verticalement au-dessus de la lette $\mathrm{M}$ du mot MYSTA. Pour les interprétations proposées de ce vase unique : Monica BAGGIO, « TITIUS EST IGITUR FICTIS CONTENDERE VERBIS QUAM PUGNARE MANU (OV. Met. XIII, 98). Il caso della pelike H3358 al Museo Archeologico di Napoli", dans Isabelle colPO, Irene FAVARETTO, Francesca FHEDINI, Iconografia 2005. Immagini et immaginari dall'Antichità classica al mondo moderno. Atti del Convegno Internazionale (Venezia, Istituto Veneto di Scienze Lettere e Arti, 26-28 gennaio 2005), Rome, Edizioni Quasar, 2006, p. 89-104.

51. Jean-Louis DURAND, « Le faire et le dire », History and Anthropology 1, 1, 1984, p. 29-48 ; version en ligne Images Re-vues [En ligne], 16|2019, mis en ligne le 01 décembre 2019. URL: http:// journals.openedition.org/imagesrevues/7308.

52. Jean-Louis DURAND, Sacrifice et Labour en Grèce ancienne. Essai d'anthropologie religieuse, Paris Rome, Éditions de la Découverte - École française de Rome, 1986, 116-123.

53. Cratère à colonnettes à f.n., Londres, British Museum B 362, 520-500 (BAPD 30320).

54. Sur les théoxénies : Michael JAMESON, «Theoxenia » dans Robin HÄGG (éd.), Ancient Greek Cult Practice from the Epigraphical Evidence, Proceedings of the Second International Seminar on Ancient Greek Cult (Athens, 22-24 November 1991), Stockholm, P. Aströms, 1994, p. 35-57 ; Louise BRUIT et François LISSARRAGUE, "Les théoxénies », ThesCRA II. 4A. Le Banquet en Grèce, 2004, p. 225-229; Gunnel EKROTH, « Meat for the Gods », dans Vinciane PIRENNE-DELFORGE et Francesca PRESCENDI, « Nourrir les dieux? " Sacrifice et représentation du divin, Actes du colloque de Liège (23-24 octobre 2009), Kernos suppl. 26, Liège, 2011, p. 15-41 ; H. collARD, « Inviter les dieux », dans ead. Montrer l'invisible, op. cit., p. 181-184.

55. Sir John Beazley les a identifiés comme les Dioscures dans ABV 338.3.

56. Sur les actes rituels aux piliers hermaïques: J.-L. DURAND, «L'Hermès multiple», op. cit.; Timothy J. MCNIVEN, «Things to which we give Service: Interactions with Sacred Images on Athenian Pottery ", dans Dimitrios YATROMANOLAKIS, An Archaeology of Representations : ancient Greek vase-painting and contemporary methodologies, Athènes, Kardamitsa, 2009, p. 298-324; H. COLLARD, « Autour du pilier hermaïque », dans ead., Montrer l'invisible, op. cit., p. 54-75.

57. Comme les images de potiers en action dans les tableaux votifs provenant du sanctuaire de Poséidon à Penteskouphia. À ce sujet voir Maria Grazia PALMIERI, Penteskouphia : immagini e parole dipinte sui pinakes corinzi dedicati a Poseidon, Athènes, Scuola archeologica italiana di Atene, 2016.

58. Comme les tableaux votifs avec sujets hippiques. Elsbeth MAUL-MANDELARTZ, Griechische Reiterdarstellungen in agonistischem Zusammenhang, Francfort, Lang, 1990.

59. K. KAROGLOU, Attic Pinakes, op. cit., p. 40-43.

60. Selon quelques rares attestations de dédicaces du type "x m'a dédié ", on constate que certains objets votifs ont leur propre voix grâce au pronom « me » utilisé.

61. Lécythe à f.r., Rome, Villa Giulia 50320, attribué au peintre du lécythe de Yale, 470-450 (BAPD 207689).

62. Pour cette série d'images voir J. GEBAUER, Pompe und Thysia, op. cit., cat. P 65-69, p. 115-118 et 181-183.

63. J. BOARDMAN et al. « Dedications. Greek dedications », op. cit., p. 270-278.

64. Vasiliki ZACHARI, «Bucrane stylisé. Au-delà de l'ornementalité » dans Gunnel EKROTH et JeanMathieu CARBON (éds.), From Snout to Tail. Exploring the Greek sacrificial animal from the literary, epigraphical, iconographical, archaeological and zooarchaeological evidence (à paraître).

65. Sur les concours de beauté voir en dernier lieu: Florence GHERCHANOC, Concours de beauté et beautés du corps en Grèce ancienne. Discours et pratiques, Bordeaux, Ausonius, 2016. 
66. Coupe à f.r., Tarquinia, Museo Nazionale Tarquiniese RC 1918, attribuée au peintre de Triptolème, vers 500 (BAPD 203880). Sur la prière: Danièle AUBRIOT-SÉVIN, Prière et conceptions religieuses en Grèce ancienne jusqu'à la fin du Ve siècle av. J.-C., Lyon, Maison de l'Orient méditerranéen, 1992 ; Simon PULLEYN, Prayer in Greek Religion, Oxford - New York, Clarendon Press - Oxford University Press, 1997; D. JAKOV, Emmanuel vouTIRAS, «Gebet, Gebärden und Handlungen des Gebetes. Das Gebet bei den Griechen », ThesCRA, 3, 2005, p. 105-141; François LISSARRAGUE, Marta PEDRINA, "Gesto, iscrizione, immagine. Attorno ad un gruppo di vasi attici a figure rosse ", dans Isabella colPO, Irene FAVARETTO et Francesca GHEDINI (éd.), Iconografia 2005 : immagini e immaginari dall'anthichità classica al mondo moderno (Actes du colloque international de l'Institut des Sciences, Lettres et Arts de Venise, 26-28 juin 2005), Rome, Quasar, 2006, p. 35-39.

67. Anthologie Palatine 9.326. Sur le culte de Nymphes: Jennifer LARSON, Greek Nymphs. Myth, Cult and Lore, Oxford, Oxford University Press, 2001.

68. Nous trouvons le même geste pour les bovidés qui sont parfois portés par les cornes avec les mains - surtout dans la série de scènes de course aux flambeaux - ou à l'aide de rênes. Dans l'iconographie dionysiaque, il faut souvent forcer le bouc en le tirant par la corne. Je remercie Stéphanie Wyler pour la précision.

69. Sur cette question voir Stella GEORGOUDI, «Le consentement de la victime sacrificielle : une question ouverte", dans Véronique MEHL et Pierre BRULÉ (éds.), Le sacrifice antique. Vestiges, procédures et stratégies, Presses Universitaires de Rennes, 2008, p. 139-153.

70. La même couleur ajoutée est utilisée pour rendre quelques détails « décoratifs » : le bouc et le cadre qui l'entoure, le méandre de la première base de la statue, le feu et les traces du sang sur l'autel, la barbe et les couronnes des hommes et du pilier hermaïque.

71. ESCHYLE, Les Suppliantes 463 ; HÉRONDAS IV 19-20 ; ARISTOPHANE, Les Thesmophories, 765-784. Pour les statues d'athlètes comme dédicaces associées à des tableaux votifs: PLINE, Histoire Naturelle 34.59 ; PAUSANIAS, V, 16, 3.

72. Franciszek sокоцошsкі, Lois sacrées de l'Asie Mineur, Paris, de Boccard, 1955, 172, n 74 ; ibid., Lois sacrées des cités grecques. Supplément, Paris, de Boccard, 1962, $\mathrm{n}^{\circ} 107,206, \mathrm{n}^{\circ} 123$, ainsi que $\mathrm{n}^{\circ}$ 43 et $\mathrm{n}^{\circ} 111$. IG II ${ }^{2} 995$; ibid., Lois sacrées des cités grecques. Supplément, Paris, de Boccard, 1969, 79, $\mathrm{n}^{\circ} 43$.

73. Amphore à f.r., Boston, Museum of Fine Arts 68.163 attribué au peintre de Nikon, 470-460 (BAPD 275790).

74. Comme des pinakes, branchages, couronnes, fruits et graines.

75. Notamment l'osphûs (la queue de l'animal sacrifié), ainsi que des fleurs, branchages, couronnes, fruits et œufs.

76. Majoritairement les pinakes et les bucranes, ainsi que d'autres objets votifs, des armes, trépieds et bandelettes.

77. Selon Pausanias (I 33, 7-8), la base en relief de cette statue est décorée de la scène de la remise d'Hélène à Léda. Il existait aussi des bases de statues peintes comme par exemple celle du monument statuaire à Aigeira: Sophie MONTEL, Recherches sur la présentation architecturale des groupes sculptés en Grèce antique, Paris, 2008 (thèse sous la direction de Bernard Holtzmann), p. 177-182.

78. Lécythe à f.n., Palerme Museo Archologico della Fondazione I. Mormino, Banco di Sicilia 2588 attribué au peintre de Thésée, vers 490 (BAPD 18489).

79. Skyphos à f.n., Salerne, Museo Nazionale P 01382, vers 500 (Para 257); Stamatis PHRITZILAS, $O$

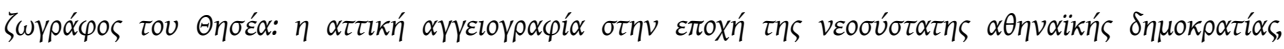

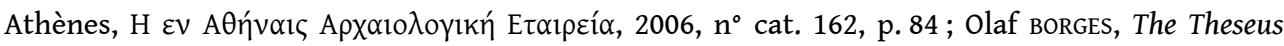
Painter. Style, shape and Iconography, Amsterdam, Allard Pierson Series, 2007, n cat. 79, p. 118-119, 154.

Images Re-vues, Hors-série 9 | 2020 


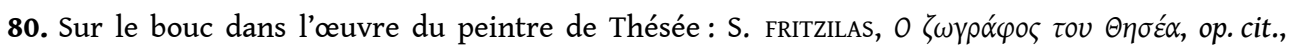
p. 38-39, 49-51, 112-115, 125-128, 222-223.

81. Athènes, Musée National 13916, vers 500 (BAPD 351584).

82. Plat à f.n., Chicago, The David and Alfred Smart Museum of Art, The University of Chicago 1967.115.256, vers 490 (BAPD 9040483).

83. Il ne s'agit pas de la seule surprise dans cette scène tout à fait originale. Pour l'oiseau voleur

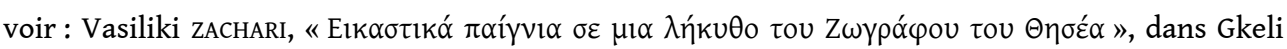

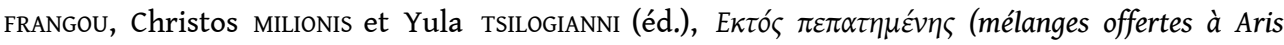
Tsaravopoulos), Athènes, Topos (à paraître).

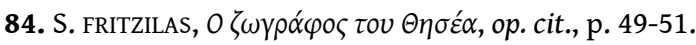

85. La même association est également attestée sur la coupe à f.r. à Berlin, Antikensammlung $F$ 2294 attribuée au peintre de la Fonderie, 490-480 (BAPD 204340). Sur la nature et la fonction de tableaux dans ce contexte plusieurs avis ont été proposés voir en détail Athina CHATZEDEMETRIOU,

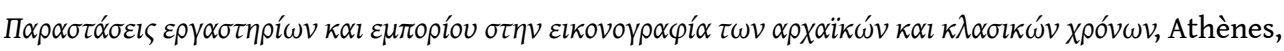
TAPA, 2005, p. 68-69.

86. Pélikè à f.r., Berlin Antikensammlung F 2172, attribué au peintre de Persée, vers 460 (BAPD 206706). Françoise FRONTISI-DUCRoux, «Une invention d'Athènes? » dans ead., Le dieu-masque. Une figure du Dionysos d'Athènes, p. 219.

87. Pierre DEVAMBEZ, "Pierres hermaïques et stèles", Revue Archéologique, 1968, p. 139-154. Lorsque ces effigies d'Hermès ont été mutilées au niveau du visage et du sexe à Athènes la veille de l'expédition en Sicile en 415, ce fut un sacrilège. L'évènement est raconté par Thucydide VI, 27-28. Sur la mutilation d'hermès : Robin OSBORNE, "The erection and mutilation of the hermai », Proceedings of the Cambridge Philological Society, 211, 1985, p.47-73; DOI: 10.1017/ S0068673500004752.

88. Voir le psykter à f.r. à Londres, British Museum E 768 attribué à Douris, vers 480 (BAPD 205309). Sur l'anatomie des satyres: François LISSARRAGUE, « De la sexualité des satyres » Mètis 1987, p. 63-90 ; ibid. « Le corps du satyres », dans ibid., La cité des satyres, Paris, éditions de l'EHESS, 2013, p. 53-72. Sur l'effet du rire des satyres : ibid., « Satyres, sérieux s'abstenir », dans Le rire des Grecs, 2000, p. 109-119.

89. Dans le seul parallèle auquel on pourrait avoir recours l'image du satyre est abîmée à cet endroit : lécythe à f.r., Londres British Museum E 585, attribué au peintre de Bowdoin, 480-470 (BAPD 208120).

90. François LISSARRAGUE, « Satyres dans la cité », dans ibid., La cité des satyres, op. cit., p. 191-215.

91. Christian MAZET, «La 'sirène' d'Orient en Occident comme exemple de la sélection culturelle des hybrides féminins en Méditerranée orientalisante ( $\mathrm{VIII}^{\mathrm{e}}-\mathrm{VI}^{\mathrm{e}}$ siècle av. J.-C.) ", dans L'animal symbole, Paris, Éditions du Comité des travaux historiques et scientifiques, 2019. En ligne : http:// books.openedition.org/cths/5065. DOI : https://doi.org/10.4000/books.cths.5065.

92. Eva HOFSTETTER Sirenen im archaïschen und klassichen Griechenland, Würzburg, K. Triltsch, 1990.

93. John BOARDMAN, « Pottery from Eretria », Annual of the British School at Athens, 47, 1952 ;

Christiane BRON, «Chouettes », Études de lettres, 6, 1983, p. 50-51.

94. Giulia Rocco, «Nyktopaidias: considerazioni sulle immagini di uccelli androcefali nell'Orientalizzante greco », Rendiconti IX, XXIV 2013 (2014), p. 289-325.

95. Herbert hoffmanN, Sexual and Asexual Pursuit, A structuralist Approach to Greek Vase Painting, Londres, Royal Anthropological Institute of Great Britain and Ireland, 1977.

96. Voir en dernier lieu la thèse de Christian MAZET, Meixoparthenoi. L'hybridité femme-animal en Méditerranée orientalisante et archaïque (VIII $-V^{e}$ s. av. J.-C.), Paris, 2020, sous la direction d'Anne Coulié et Stéphane Verger.

97. Gaëlle DESCHODT, «Sirènes et mariage dans la céramique attique », dans Anna CAIOzzo (éd.), Femmes médiatrices et ambivalentes. Mythes et imaginaires. Paris, Armand Colin, «Recherches », 
2012, p. 307-322. DOI : 10.3917/arco.caioz.2012.01.0307. URL : https://www.cairn.info/femmesmediatrices-et-ambivalentes--9782200272814-page-307.htm.

98. Jean-Pierre VERNANT, «Hestia-Hermès. Sur l'expression religieuse de l'espace et du mouvement chez les Grecs ", dans ibid., Mythe et pensée chez les Grecs, Paris, La Découverte, p. 157-158. Sur Hermès comme passeur: Laurence KAHN, Hermès passe ou les ambiguités de la communication, Paris, Maspero, 1978 ; et dans l'iconographie J.-L. DURAND, «L'Hermès multiple ", op. cit.

99. Pierre-Olivier DITTMAR, Pierre-Antoine FABRE, Thomas GOLSENNE, Caroline PERRÉE, Matérialiser les désirs. Techniques votives Techniques \& Culture, 70 | 2018 DOI : https://doi.org/10.4000/tc.9361.

100. Dans une autre variation la croix dans le pinax est combinée avec quatre points plus épais sur un cratère à calice à f.r., Londres British Museum F 9, vers 400 (BAPD 18503).

101. Comme par exemple dans le lécythe à figures rouges à Palerme (fig. 7).

102. Ce jeu d'autoréférentialité à la production de la céramique est plus explicite sur les vases dont les scènes représentent les activités dans l'atelier du potier. Pour l'univers romain, voir l'article de Stéphanie Wyler : https://journals.openedition.org/imagesrevues/7967.

103. À propos de ce motif et des jeux d'images suscités voir la contribution de Françoise FrontisiDucroux dans le dossier : https://journals.openedition.org/imagesrevues/8852.

104. Cratère à f.r., Naples, Museo Archeologico Nazionale 82599, attribué au peintre de Rodin 966 , vers 400 (BAPD 218178) où il y a cinq pinakes et un bucrane. Ces images de format horizontal ne sont pas à confronter avec les écritoires qui sont peints dans le champ de l'image souvent avec le stylet comme par exemple sur le fragment de chous à f.r., Athènes Musée de l'Agora $\mathrm{P}$ 18286, 420-400 (BAPD 3410).

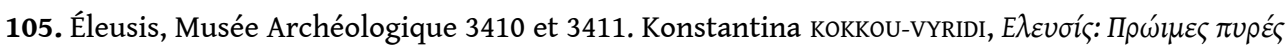

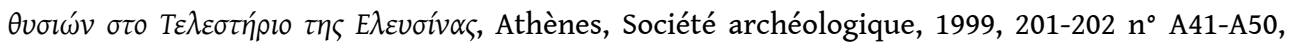
pl. 10-11; K. KAROGLOU, Attic Pinakes, op. cit., $\mathrm{n}^{\circ}$ cat. 141 et 142, p. 102-103.

106. Itzhak GOLDBERG, « Avant-propos », dans ibid. (éd.), L'art du vide, Paris, CNRS éditions, 2017, p. 15.

107. Ce dossier est étudié dans le cadre de la thèse de V. ZACHARI, Autour de l'autel... op. cit.

108. Jean-Louis DURAND et François LISSARRAGUE, "Un lieu d'image? L'espace du loutérion », Hephaistos 2, 1980, p. 89-106.

109. Vasiliki ZACHARI, «Espace vide: le degré zéro de l'image?», dans Vasiliki ZACHARI, Élise LEHOUX \& Noémie Hosoi, La cité des regards. Autour de F. Lissarrague, Rennes, Presses universitaires de Rennes, 2019, p. 257-268.

110. Tonio HÖLSCHER, La vie des images grecques. Sociétés de statues, rôles d'artistes et notions esthétiques dans l'art grec ancienne, Paris, Louvre éditions / Hazan, 2015, p. 78.

111. Sur les formules de dédicace et de fondation d'autels et piliers hermaïques : J. BOARDMAN et al. « Dedications. Greek dedications », op. cit.

112. François LISSARRAGUE, «Figuring religious rituals », dans Tyler Jo SMITH, Dimitris PLANTZos, $A$ Companion to Greek Art, Oxford, Blackwell, 2012, vol. 2, p. 572.

\section{RÉSUMÉS}

Les tableaux votifs, dit pinakes en grec ancien, étaient parmi les offrandes les plus communes. Ils représentent des divinités, des scènes mythologiques et rituelles, ainsi que des dédicants. Quant à 
la représentation de pinakes sur les vases, les images ne sont pas en accord avec les realia archéologiques. Les peintres s'inspirent de leurs propres productions pour dessiner les motifs dans les pinakes créant ainsi des jeux d'images avec la scène principale. Ainsi, l'image du pilier hermaïque, d'une figure humaine, du bouc, des êtres hybrides, mais aussi les motifs abstraits, comme l'absence de figuration sont à chaque fois porteurs de significations et d'effets visuels originaux dans l'ensemble de l'image.

The so called pinakes in ancient Greek (votive images) were amongst the most common offerings. Divinities, mythological and ritual scenes, as well as the dedicators figured on them. As for the depiction of pinakes on vases, the images do not match with the archaeological realia. Painters are inspired by their own productions in order to draw the motifs on the pinakes creating pictorial games with the main scene. Thus, the image of a herm, human figure, billy goat, as well as of abstract motifs or even the absence of any figuration bear original significations and visual effects in the whole scene.

\section{INDEX}

Mots-clés : Vase attiques, pinakes, tableaux votifs, mise en abyme, autoréférentialité

Keywords : Attic vases, pinakes, votive paintings, mise en abyme, self-referentiality

\section{AUTEUR}

\section{VASILIKI ZACHARI}

Vasiliki Zachari est diplômée en Archéologie et Histoire de l'Art (Université d'Athènes). Sa thèse porte sur les représentations de l'autel dans la céramique attique $\mathrm{du} \mathrm{VI}^{\mathrm{e}}$ et du ve s. av. J.-C. (sous la direction de F. Lissarrague à l'EHESS, centre ANHIMA). Membre du comité de rédaction d'Images $R e$-vues depuis 2009, elle a également coédité l'ouvrage collectif La cité des regards. Autour de François Lissarrague, Rennes, PUR, 2019 (avec É. Lehoux et N. Hosoi) et elle a édité le numéro Gestualité du rituel d'Images Re-vues, 2019. 